\title{
Optically induced forces and torques: Interactions between nanoparticles in a laser beam
}

\author{
David S. Bradshaw and David L. Andrews \\ Nanostructures and Photomolecular Systems, School of Chemical Sciences, University of East Anglia, \\ Norwich, NR4 7TJ, United Kingdom \\ (Received 15 April 2005; published 16 September 2005)
}

\begin{abstract}
Distinctive optical forces and torques arise between nanoparticles irradiated by intense laser radiation. These forces, associated with a pairwise process of stimulated scattering, prove to enable the possibility of producing significant modifications to both the form and magnitude of interparticle forces, with additional contributions arising in the case of dipolar materials. Moreover, such forces have the capacity to generate unusual patterns of nanoscale response, entirely controlled by the input beam characteristics-principally the optical frequency, intensity, and polarization. Based on quantum electrodynamical theory, a general result is secured for the laser-induced force under arbitrary conditions, incorporating both static and dynamic coupling mechanisms. Specific features of the results are identified for pairs of particles with prolate cylindrical symmetry, e.g., carbon nanotubes, where it is shown that the laser-induced forces and torques are sensitive functions of the pair spacing and orientation, and the laser beam geometry; significantly, they can be either repulsive or attractive according to conditions. For nanoparticles trapped in a Laguerre-Gaussian laser beam the results also reveal additional and highly distinctive torques that suggest further possibilities for nanomanipulation with light. The paper concludes with a discussion on several potential applications of such forces.
\end{abstract}

DOI: 10.1103/PhysRevA.72.033816

\section{INTRODUCTION}

In the early years of laser development, new possibilities began to emerge for the practical utilization of optomechanical forces to manipulate small particles, largely owing to the pioneering work of Ashkin [1]. The field evolved rapidly and by the mid-1980s it had led to the invention of optical tweezers [2], a technique which has since become a mainstream tool for the optical trapping and manipulation [3-6] of a diverse range of particles, from living cells down to single atoms. More recently, alongside burgeoning biological applications, there has been a growing recognition of the potential for other distinctive nanotechnological applications of laserinduced forces. The activity in this area has seen a huge increase in its range, with the latest advances leading to applications such as microviscometry $[7,8]$. There is also considerable interest in the deployment of exquisite new beam structures for laser light $[9,10]$. Notably, the use of "twisted" Laguerre-Gaussian (LG) beams in optical tweezers can produce effects that have become known as "optical spanners," that can not only trap particles but also rotate them-a result of angular momentum transfer [11,12]. Indeed it has been shown that a superposition of LG beams can simultaneously trap numerous particles in a three-dimensional array and, additionally, control their rotations $[13,14]$.

Optical trapping and manipulation is generally based on particles experiencing a radiative attraction towards a highintensity region of a laser beam. The motion of two or more particles, subject to these optomechanical forces within the beam, is also influenced by interparticle forces. The fundamental character of such forces is very well known and, for particles separated beyond the region of wave-function overlap, derives from dipole interactions, dispersion forces, etc. Quantum mechanics provides the framework for their detailed evaluation. However, it has recently emerged [15]
PACS number(s): 42.50.Ct, 32.80.Lg, 34.20.Gj, 85.85.+j

from studies based on quantum electrodynamics (QED) - a theory that designedly addresses the quantum interactions of matter with radiation - that the throughput of intense laser light can significantly modify the form and magnitude of interparticle coupling forces. As detailed in this paper, the mechanism for these optically induced pair interactions is one of stimulated scattering. Thus, entirely distinct and separable from the optomechanical interactions involved in optical tweezers, laser-induced interparticle forces and torques are capable of generating interesting patterns of particle motion determined by the intensity, polarization, and other features of the laser input.

First identified in a definitive treatment by Thirunamachandran [16], the concept of laser-induced coupling forces has received attention from several theory research groups [17-22], was cited in a prominent futurology of chemistry [23] and has been made the subject of experimental investigation $[24,25]$. It is noteworthy that, since its original postulation, the threshold levels of intensity necessary to induce significant forces (typically megawatts per square centimeter) have become routinely available-for example by focusing the output of a standard titanium:sapphire femtosecond laser. Accordingly, the potential significance of the subject has soared in importance, and applications to the optical control of Bose-Einstein condensates have already been envisaged [26-30]. In this paper we use a quantum electrodynamical approach to determine complete and general results for optically induced forces between chemically identical particles, also applying the results to a number of systems of current interest.

In Sec. II a general expression is first derived for particles of arbitrary shape and electronic characteristics. This is followed by a detailed analysis of two systems which differ in the angular disposition of individual particles against the incoming laser light; specifically, where parallel particles of cylindrical symmetry are disposed at a variable angle to the electric field vector of the incident light (Sec. III A), and 
where the particles can tumble freely with respect to the field vector (Sec. III B). A nanoparticle pair irradiated by LG beam (or any other beam endowed with "orbital" angular momentum) can give rise to additional angular features. Employing a recently completed QED formulation of the corresponding interaction with matter [31], Sec. IV presents an analysis that leads to an expression for the torque operating between nanoparticles irradiated with such "twisted" radiation. Section V addresses the applications of laser-induced forces, including carbon nanotubes, nanoelectromechanical systems, and Bose-Einstein condensates. The paper concludes with a summary.

\section{GENERAL DERIVATION}

The framework of QED [32] —in which both radiation and matter are treated quantum mechanically-provides a highly satisfactory theory for the study of optically induced nanoparticle interactions. Using the Power-Zienau-Woolley approach [33], the full Hamiltonian $H$, for a pair of particles $A$ and $B$ is given by

$$
H=\sum_{\xi=A, B} H_{\mathrm{mol}}(\xi)+\sum_{\xi=A, B} H_{\mathrm{int}}(\xi)+H_{\mathrm{rad}},
$$

where $H_{\mathrm{mol}}^{\xi}$ is the multipolar Hamiltonian for particle $\xi$ and $H_{\text {rad }}$ denotes the radiation field. The operator $H_{\text {int }}^{\xi}$ represents the interaction of the field with $\xi$ and, in the electric-dipole approximation, is given by

$$
H_{\mathrm{int}}^{\xi}=-\varepsilon_{0}^{-1} \sum_{\xi} \boldsymbol{\mu}(\xi) \cdot \mathbf{d}^{\perp}\left(\mathbf{R}_{\xi}\right),
$$

with $\boldsymbol{\mu}(\xi)$ and $\mathbf{R}_{\xi}$, respectively, denoting the electric-dipole moment operator and the position vector of a dielectric nanoparticle labeled $\xi$. Note that, in any case where the nanoparticles comprise individual, electronically distinct components such as color centers, chromophores, or molecules, $\boldsymbol{\mu}(\xi)$ is to be interpreted as an effective dipole operator-comprising the sum of dipole operators for each of the constituent units. This is justified on the basis that the dipole operator entails a sum over all charges, and in such a case these are attributable to individual units. Returning to Eq. (2.2), the operator $\mathbf{d}^{\perp}\left(\mathbf{R}_{\xi}\right)$ represents the transverse electric displacement field, expressible in a mode expansion involving summations over all wave vectors $\mathbf{p}$ and polarizations $\varepsilon$,

$$
\begin{aligned}
\mathbf{d}^{\perp}\left(\mathbf{R}_{\xi}\right)= & i \sum_{\mathbf{p}, \varepsilon}\left(\frac{\hbar c p \varepsilon_{0}}{2 V}\right)^{1 / 2}\left[\mathbf{e}^{(\varepsilon)}(\mathbf{p}) a^{(\varepsilon)}(\mathbf{p}) e^{i\left(\mathbf{p} \cdot \mathbf{R}_{\xi}\right)}-\overline{\mathbf{e}}^{(\varepsilon)}(\mathbf{p})\right. \\
& \left.\times a^{\dagger(\varepsilon)}(\mathbf{p}) e^{-i\left(\mathbf{p} \cdot \mathbf{R}_{\xi}\right)}\right] .
\end{aligned}
$$

Here $\mathbf{e}^{(\varepsilon)}(\mathbf{p})$ is the polarization unit vector $\left[\overline{\mathbf{e}}^{(\varepsilon)}(\mathbf{p})\right.$ being its complex conjugate, the admission of complex polarizations allowing for circular or elliptical as well as plane polarization], $V$ is an arbitrary quantization volume, and $a^{(\varepsilon)}(\mathbf{p}), a^{\dagger(\varepsilon)}(\mathbf{p})$ are, respectively, the photon annihilation and creation operators for a radiation mode $(\mathbf{p}, \varepsilon)$.

To secure a general result for the force between the pair of particles a suitable starting point is to derive an expression for the energy shift $\Delta E$. The latter can be obtained by the

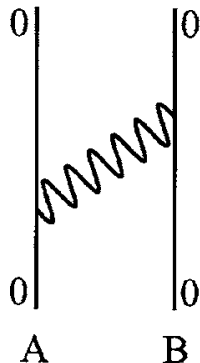

(a)

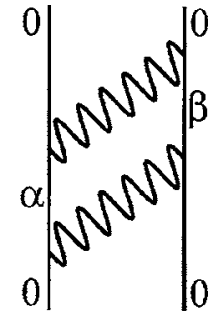

A

(b)
FIG. 1. (a) One of two Feynman diagrams for the coupling of two static dipole moments: 0 denotes the ground state. In the other diagram the virtual photon propagates from $B$ to $A$. (b) One of 12 Feynman diagrams [32] used as a calculational aid in determination of the dispersion energy; $\alpha$ and $\beta$ are virtual excited states for particles $A$ and $B$, respectively.

application of perturbation theory, and the leading terms are as follows:

$$
\begin{aligned}
\Delta E= & \operatorname{Re}\left(\sum_{r} \frac{\left\langle i\left|H_{\text {int }}\right| r\right\rangle\left\langle r\left|H_{\text {int }}\right| i\right\rangle}{\left(E_{i}-E_{r}\right)}\right. \\
& \left.+\sum_{t, s, r} \frac{\left\langle i\left|H_{\text {int }}\right| t\right\rangle\left\langle t\left|H_{\text {int }}\right| s\right\rangle\left\langle s\left|H_{\text {int }}\right| r\right\rangle\left\langle r\left|H_{\text {int }}\right| i\right\rangle}{\left(E_{i}-E_{t}\right)\left(E_{i}-E_{s}\right)\left(E_{i}-E_{r}\right)}\right),
\end{aligned}
$$

where $|i\rangle$ is the unperturbed state in which both particles are in their electronic ground state, $|r\rangle,|s\rangle$, and $|t\rangle$ are virtual states, and $E_{n}$ is the energy of state $|n\rangle$. The last signifies one of the basis states for the perturbative development, expressible in the form

$$
|n\rangle=\left|\operatorname{mol}_{n}\right\rangle\left|\operatorname{rad}_{n}\right\rangle \equiv\left|\operatorname{mol}_{n} ; \operatorname{rad}_{n}\right\rangle,
$$

with $\left|\mathrm{mol}_{n}\right\rangle$ and $\left|\mathrm{rad}_{n}\right\rangle$ defining the status of all particles and radiation states, respectively. In Eq. (2.4), each operation of $H_{\text {int }}$ on the state to its right effects a transition to the state on its left-physically this must signify the annihilation or creation of a photon, as follows from Eqs. (2.2) and (2.3).

One interaction that is determined by second-order perturbation theory - the first term of Eq. (2.4)-is the coupling of two static (permanent) dipoles, which in the QED formulation involves the creation of a virtual photon at one particle and its annihilation at the other (Fig. 1(a)). Another familiar form of interaction is responsible for the dispersion energy; this entails four photon-particle coupling events through the creation and annihilation of two virtual photons [32] (Fig. $1(\mathrm{~b}))$. Here $\Delta E$ is accordingly determined by fourth-order perturbation theory and emerges from the second term of Eq. (2.4). In the present context, however, it is more important to note that this fourth-order term also accommodates a form of interaction that can only arise through coupling with throughput radiation, the focus of the work that follows. The detailed representation of this mechanism using the Feynman diagrammatic method necessitates the construction of 96 time-ordered diagrams, four of which are shown in Figs. 2 and 3. In general this laser-induced interaction involves $\Delta E_{\text {ind }}$ contributions of two kinds: in one, the annihilation of a "real" input photon at one particle and the stimulated emis- 


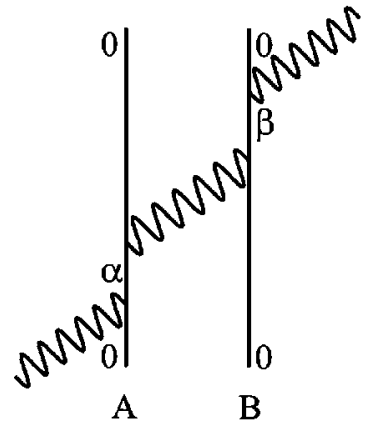

(a)

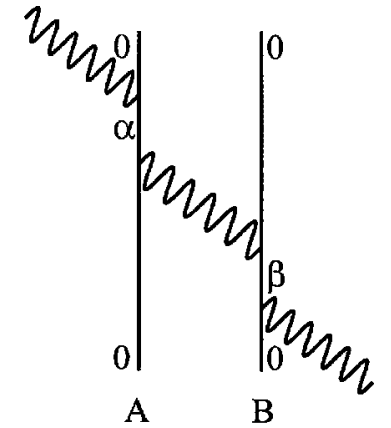

(b)
FIG. 2. Two typical Feynman diagrams (each with 23 further permutations) for calculation of dynamic contributions to the laserinduced interaction energy.

sion of an equivalent "real" photon at the other is effected through virtual photon mediation between the two particles (Fig. 2) - this is a dynamic form of coupling, so called because a real and finite amount of energy is conveyed between the two particles. In the other contribution to $\Delta E_{\text {ind }}$, the photon creation and annihilation events both occur at the same particle, again with interparticle coupling mediated by a virtual photon (Fig. 3) - which represents a static form of coupling, with no net conveyance of energy between the particles. In each case the particles and throughput radiation suffer no overall change in state. Here, a real photon denotes a quantum of electromagnetic radiation with a long lifetime and commonly termed real characteristics-although physically, there is a sense in which all photons are virtual in character [34].

Although the construction of a complete set of Feynman graphs can expedite the development of a result for the laserinduced energy shift, a more recently devised state-sequence method [35] proves to offer considerable advantages. Specifically, all 96 time orderings are accommodated in just four state-sequence diagrams - which also offer a more direct calculational route to the result (see the Appendix). Two examples are shown in Figs. 4 and 5. The explicit result for $\Delta E_{\text {ind }}$ follows insertion of Eqs. (2.2) and (2.3) into the second term of Eq. (2.4), recognizing that $\boldsymbol{\mu}(\xi)$ and $\mathbf{d}^{\perp}\left(\mathbf{R}_{\xi}\right)$ operate on $\left|\mathrm{mat}_{n}\right\rangle$ and $\left|\operatorname{rad}_{n}\right\rangle$, respectively - the latter through

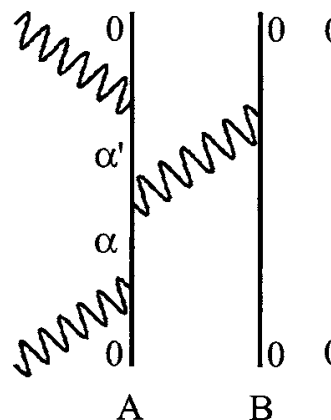

(a)

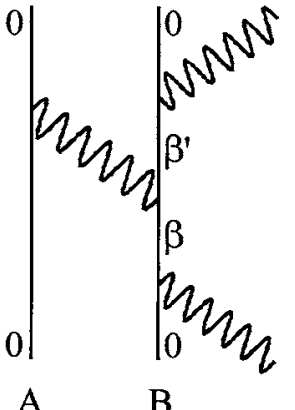

(b)
FIG. 3. Two Feynman diagrams (each with 23 further permutations) for calculation of static contributions to the laser-induced interaction energy.

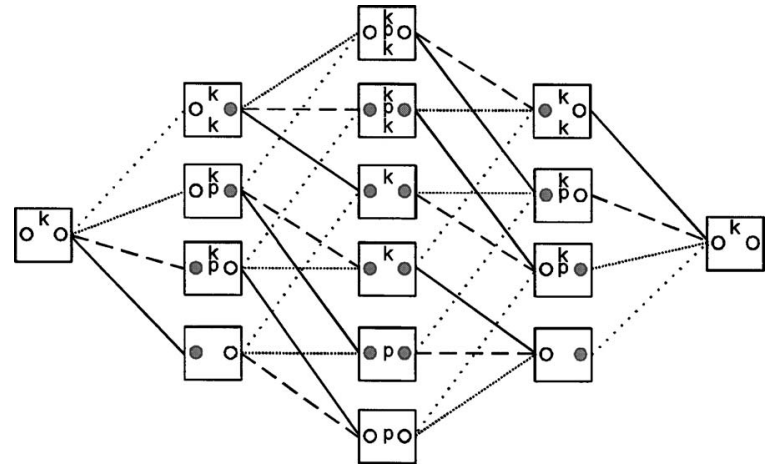

FIG. 4. One of two state-sequence diagrams for calculation of the dynamic contributions to the laser-induced interaction, associated with the annihilation of an input photon at $A$ and creation of an output photon at $B$. Each of the 24 continuous channels across the diagram from left to right corresponds to a distinct Feynman graph. Key: $k$, real photon; $p$, virtual photon; open circles, particle in ground state (where particle $A$ is a circle on the left side of the box and $B$ is on the right); gray circles, particle in virtual excited state; solid lines, real photon annihilation at $A$; dashed lines, virtual photon coupling event at $A$; closed-spaced dotted lines, virtual photon coupling at $B$; wide-spaced dotted lines, real photon creation at $B$.

the following relationships $a^{(\varepsilon)}(\mathbf{p})|n(\mathbf{p}, \varepsilon)\rangle=\sqrt{n}|(n-1)(\mathbf{p}, \varepsilon)\rangle$ and $a^{\dagger(\varepsilon)}(\mathbf{p})|n(\mathbf{p}, \varepsilon)\rangle=\sqrt{n+1}|n+1(\mathbf{p}, \varepsilon)\rangle$. Therefore, by the same perturbation method as used in previous work [36], the following concise result emerges;

$$
\begin{aligned}
\Delta E_{\text {ind }}= & \left(\frac{n \hbar c k}{\varepsilon_{0} V}\right) \operatorname{Re}\left\{e_{i}^{(\lambda)} \bar{e}_{l}^{(\lambda)} \alpha_{i j}^{A}(k) V_{j k}(k, \mathbf{R}) \alpha_{k l}^{B}(k) \exp (i \mathbf{k} \cdot \mathbf{R})\right. \\
& \left.+\frac{1}{2} e_{i}^{(\lambda)} \bar{e}_{l}^{(\lambda)} V_{j k}(0, \mathbf{R})\left[\beta_{i j l}^{A}(k) \mu_{k}^{B}+\mu_{k}^{A} \beta_{i j l}^{B}(k)\right]\right\} .
\end{aligned}
$$

Here, use is made of the implied summation convention for repeated Cartesian tensor indices; $\mu_{k}^{\xi}$ is a static (permanent) dipole moment and, as defined below, $\alpha_{i j}^{\xi}(k)$ is the dynamic polarizability tensor, and $\beta_{i j k}^{\xi}(k)$ is a linear electro-optic hyperpolarizability tensor [37]. Also in Eq. (2.6) $\mathbf{k}$ and $\hbar c k$ denote the input wave vector and photon energy, respec-

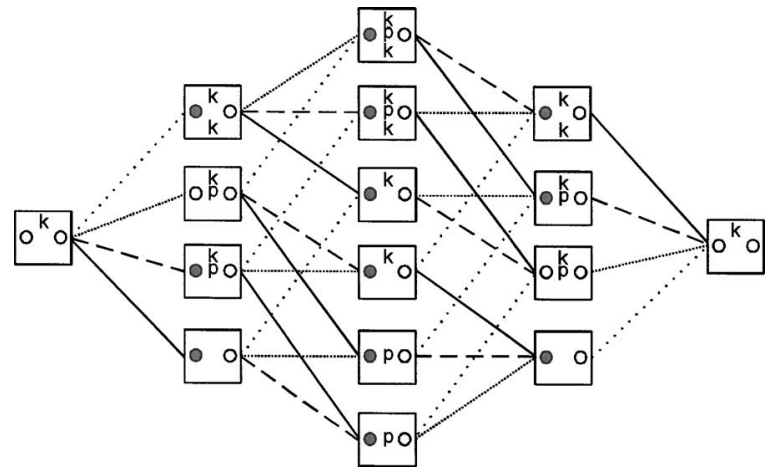

FIG. 5. One of two state-sequence diagrams for calculation of the static contributions to the laser-induced interaction, associated with both the annihilation of an input photon and the creation of an output photon at $A$. For key, see caption of Fig. 4, but here the wide-spaced dotted lines indicate real photon creation at $A$. 
tively, and $V_{j k}$ signifies the fully retarded resonance electricdipole-electric-dipole interaction tensor of the general form [38]

$$
\begin{aligned}
V_{i j}(k, \mathbf{R})= & \frac{e^{i k R}}{4 \pi \varepsilon_{0} R^{3}}\left[(1-i k R)\left(\delta_{i j}-3 \hat{R}_{i} \hat{R}_{j}\right)\right. \\
& \left.-(k R)^{2}\left(\delta_{i j}-\hat{R}_{i} \hat{R}_{j}\right)\right],
\end{aligned}
$$

which is dependent on the interparticle displacement vector $\mathbf{R} \equiv \mathbf{R}_{B}-\mathbf{R}_{A}$. Returning to the optical response tensors, these are explicitly given by the following:

$$
\alpha_{i j}^{\xi}(k)=\sum_{s}\left(\frac{\mu_{i}^{i s} \mu_{j}^{s i}}{E_{s i}-\hbar c k-i \Gamma_{s}}+\frac{\mu_{j}^{i s} \mu_{i}^{s i}}{E_{s i}+\hbar c k-i \Gamma_{s}}\right)
$$

and

$$
\begin{aligned}
\beta_{i j k}^{\xi}(k)= & \sum_{s, t}\left(\frac{\mu_{i}^{0 t} \mu_{j}^{t s} \mu_{k}^{s 0}}{\left(E_{t 0}-\hbar c k-i \Gamma_{t}\right)\left(E_{s 0}-\hbar c k-i \Gamma_{s}\right)}\right. \\
& +\frac{\mu_{i}^{0 t} \mu_{k}^{t s} \mu_{j}^{s 0}}{\left(E_{t 0}-\hbar c k-i \Gamma_{t}\right)\left(E_{s 0}-i \Gamma_{s}\right)} \\
& +\frac{\mu_{j}^{0 t} \mu_{i}^{t s} \mu_{k}^{s 0}}{\left(E_{t 0}-i \Gamma_{t}\right)\left(E_{s 0}-\hbar c k-i \Gamma_{s}\right)} \\
& +\frac{\mu_{k}^{0 t} \mu_{i}^{t s} \mu_{j}^{s 0}}{\left(E_{t 0}+\hbar c k-i \Gamma_{t}\right)\left(E_{s 0}-i \Gamma_{s}\right)} \\
& +\frac{\mu_{j}^{0 t} \mu_{k}^{t s} \mu_{i}^{s 0}}{\left(E_{t 0}-i \Gamma_{t}\right)\left(E_{s 0}+\hbar c k-i \Gamma_{s}\right)} \\
& \left.+\frac{\mu_{k}^{0 t} \mu_{j}^{t s} \mu_{i}^{s 0}}{\left(E_{t 0}+\hbar c k-i \Gamma_{t}\right)\left(E_{s 0}+\hbar c k-i \Gamma_{s}\right)}\right),
\end{aligned}
$$

respectively, where $\boldsymbol{\mu}^{x y}=\langle x|\boldsymbol{\mu}| y\rangle, E_{x y}=E_{x}-E_{y}$, and $\Gamma_{s}, \Gamma_{t}$ are damping factors.

Returning to Eq. (2.6) it is to be noted that, by simple index symmetry arguments, the first term accommodates contributions in which the roles of $A$ and $B$ in the dynamic mechanism interchange (see the Appendix), whereas the subsequent terms differentiate the roles of $A$ and $B$ in the static mechanism. The result is thus cast in a form that allows consideration of cases where $A$ and $B$ are either identical or dissimilar. Next, from Eq. (2.6) the laser-induced force $\mathbf{F}_{\text {ind }}$ $=-\partial \Delta E_{\text {ind }} / \partial \mathbf{R}$ emerges as follows:

$$
\begin{aligned}
F_{m}= & \left(\frac{I}{8 \pi \varepsilon_{0}^{2} c R^{4}}\right)\left(2 \operatorname{Re}\left[e_{i}^{(\lambda)} \bar{e}_{l}^{(\lambda)} \alpha_{i j}^{\mathrm{A}}(k) \alpha_{k l}^{\mathrm{B}}(k)\right]\right. \\
& \times\left\{-k^{3} R^{3}\left(\cos k R \sin (\mathbf{k} \cdot \mathbf{R}) \hat{k}_{m}+\sin k R \cos (\mathbf{k} \cdot \mathbf{R}) \hat{R}_{m}\right)\right. \\
& \times\left(\delta_{j k}-\hat{R}_{j} \hat{R}_{k}\right)-k^{2} R^{2}\left[\operatorname { c o s } k R \operatorname { c o s } ( \mathbf { k } \cdot \mathbf { R } ) \left(2 \delta_{j k} \hat{R}_{m}+\delta_{m j} \hat{R}_{k}\right.\right. \\
& \left.\left.+\delta_{m k} \hat{R}_{j}-6 \hat{R}_{j} \hat{R}_{k} \hat{R}_{m}\right)-\hat{k}_{m} \sin k R \sin (\mathbf{k} \cdot \mathbf{R})\left(\delta_{j k}-3 \hat{R}_{j} \hat{R}_{k}\right)\right] \\
& +k R\left[3 \operatorname { s i n } k R \operatorname { c o s } ( \mathbf { k } \cdot \mathbf { R } ) \left(\delta_{j k} \hat{R}_{m}+\delta_{m j} \hat{R}_{k}+\delta_{m k} \hat{R}_{j}\right.\right. \\
& \left.\left.-5 \hat{R}_{j} \hat{R}_{k} \hat{R}_{m}\right)+\hat{k}_{m} \cos k R \sin (\mathbf{k} \cdot \mathbf{R})\left(\delta_{j k}-3 \hat{R}_{j} \hat{R}_{k}\right)\right] \\
& +3 \cos k R \cos (\mathbf{k} \cdot \mathbf{R})\left(\delta_{j k} \hat{R}_{m}+\delta_{m j} \hat{R}_{k}+\delta_{m k} \hat{R}_{j}\right.
\end{aligned}
$$

$$
\begin{aligned}
& \left.\left.-5 \hat{R}_{j} \hat{R}_{k} \hat{R}_{m}\right)\right\}+3 \operatorname{Re}\left\{e_{i}^{(\lambda)} \bar{e}_{l}^{(\lambda)}\left[\beta_{i j l}^{\mathrm{A}}(k) \mu_{k}^{\mathrm{B}}+\beta_{i j l}^{\mathrm{B}}(k) \mu_{k}^{\mathrm{A}}\right]\right\} \\
& \left.\times\left(\delta_{j k} \hat{R}_{m}+\delta_{m j} \hat{R}_{k}+\delta_{m k} \hat{R}_{j}-5 \hat{R}_{j} \hat{R}_{k} \hat{R}_{m}\right)\right),
\end{aligned}
$$

where $I=n \hbar c^{2} k / V$ is the input irradiance. One of the key developments departing from the classical result [19] is the inclusion in Eq. (2.10) of terms associated with the static coupling mechanism, as well as the dynamic terms. Also of significance is that, when Eq. (2.10) is applied to specific cases, it reduces to the dynamic term in the classical representation. The full QED expression is more general, in the following respects: (i) the polarizability is allowed to be anisotropic and dynamic, in the sense that its frequency dependence is explicit; (ii) the input wave vector can have arbitrary orientations with respect to the interparticle displacement vector; and (iii) the input radiation is also polarized arbitrarily. In passing we note that, although a general expression identical to Eq. (2.10) could in principle have been determined with the employment of classical electrodynamics, QED proves more directly amenable for this calculationand it offers additional insights into the underlying physics.

Before proceeding further, the inclusion and significance of static terms deserves comment. Whereas particles that possess a permanent static moment $\boldsymbol{\mu}$ necessarily admit nonzero components of the $\boldsymbol{\beta}$ tensor, the presence of nonzero $\boldsymbol{\beta}$ components is not a sufficient condition for the existence of $\boldsymbol{\mu}$. Specifically, for a nonzero $\boldsymbol{\mu}$ it is necessary that an axial vector possesses one or more components that transform under the totally symmetric representation of the appropriate point group, whereas for the $\boldsymbol{\beta}$ tensor the less restrictive condition is that any one or more of the irreducible weights spanning the interval $(0,3)$, resulting from decomposition of the reducible tensor, transforms under the appropriate totally symmetric representation. In particles of $\overline{6} m 2\left(D_{3 h}\right)$ symmetry, for example, a $\boldsymbol{\beta}$ tensor is allowed whereas a static dipole is not-for a full tabulation of the possibilities, see Appendix 6 in Ref. [39]. This feature is particularly significant in connection with forces between dissimilar particles, only one of which is polar, since in this case the static coupling mechanism has a level of significance similar to the dynamic mechanism. In the case of coupling between two polar particles, $\boldsymbol{\mu}-\boldsymbol{\mu}$ electrostatic interactions, i.e., coupling of two static (permanent) dipoles, will of course greatly outweigh both $\boldsymbol{\alpha}-\boldsymbol{\alpha}$ and $\boldsymbol{\mu}-\boldsymbol{\beta}$ interactions.

\section{NANOPARTICLES OF CYLINDRICAL SYMMETRY}

\section{A. Parallel particles}

The first system to be examined in detail is a pair of nonpolar nanoparticles of cylindrical symmetry, aligned in parallel along the $X$ axis of a laboratory frame, both perpendicular to $\mathbf{R}$ - the latter identified with the $Z$ axis. Planepolarized throughput radiation is defined through the angles $\phi$ and $\theta$ made by its $\mathbf{e}$ vector against $\mathbf{R}$ in the $X Z$ plane, and the particle axis in the $X Y$ plane, respectively (Fig. 6). Only the first term of Eq. (2.6) needs to be considered which, by expressing the polarization unit vector in the cylindrical form $\mathbf{e}=\sin \phi \cos \theta \mathbf{i}+\sin \phi \sin \theta \mathbf{j}+\cos \phi \mathbf{k}$, and defining the whole system in terms of the laboratory frame, gives 


$$
\begin{aligned}
\Delta E_{\text {ind }}= & \left(\frac{I}{\varepsilon_{0} c}\right) \operatorname{Re}\left(\sum_{J, K} \sin ^{2} \phi \cos ^{2} \theta \alpha_{Z J}^{\mathrm{A}} V_{J K} \alpha_{K Z}^{\mathrm{B}}\right. \\
& -\sin ^{2} \phi \sin \theta \cos \theta \alpha_{Z J}^{\mathrm{A}} V_{J K} \alpha_{K Y}^{\mathrm{B}} \\
& -\sin \phi \cos \phi \cos \theta \alpha_{Z J}^{\mathrm{A}} V_{J K} \alpha_{K X}^{\mathrm{B}} \\
& -\sin ^{2} \phi \sin \theta \cos \theta \alpha_{Y J}^{\mathrm{A}} V_{J K} \alpha_{K Z}^{\mathrm{B}}+\sin ^{2} \phi \sin ^{2} \theta \\
& \times \alpha_{Y J}^{\mathrm{A}} V_{J K} \alpha_{K Y}^{\mathrm{B}}+\sin \phi \cos \phi \sin \theta \alpha_{Y J}^{\mathrm{A}} V_{J K} \alpha_{K X}^{\mathrm{B}} \\
& -\sin \phi \cos \phi \cos \theta \alpha_{X J}^{\mathrm{A}} V_{J K} \alpha_{K Z}^{\mathrm{B}} \\
& +\sin \phi \cos \phi \sin \theta \alpha_{X J}^{\mathrm{A}} V_{J K} \alpha_{K Y}^{\mathrm{B}} \\
& \left.+\cos ^{2} \phi \alpha_{X J}^{\mathrm{A}} V_{J K} \alpha_{K X}^{\mathrm{B}}\right) \cos (\mathbf{k} \cdot \mathbf{R})
\end{aligned}
$$

where the $k$ and $\mathbf{R}$ dependence are henceforth suppressed and the indices $I, J$, and $K$ are in the laboratory frame. Employing the explicit form of the $V_{J K}$ tensor-i.e., the expression of (2.7), also identifying the unit vector $\hat{\mathbf{R}}$ as pointing in the $Z$ direction-and, for each cylindrical particle [40] $\alpha_{Y Y}$ $=\alpha_{Z Z}=\alpha_{\perp}$ and $\alpha_{X X}=\alpha_{\|}$, Eq. (3.1) is written as

$$
\begin{aligned}
\Delta E_{\mathrm{ind}}= & \left(\frac{I}{4 \pi \varepsilon_{0}^{2} c}\right)\left[\left(\alpha_{\perp}^{2} \sin ^{2} \phi \sin ^{2} \theta+\alpha_{\|}^{2} \cos ^{2} \phi\right)\right. \\
& \times\left(\frac{\cos k R}{R^{3}}+\frac{k \sin k R}{R^{2}}-\frac{k^{2} \cos k R}{R}\right) \\
& \left.-2 \alpha_{\perp}^{2} \sin ^{2} \phi \cos ^{2} \theta\left(\frac{\cos k R}{R^{3}}+\frac{k \sin k R}{R^{2}}\right)\right] \cos (\mathbf{k} \cdot \mathbf{R}) .
\end{aligned}
$$

Here, the superscripts $A$ and $B$ are omitted as the two particles are identical. The laser-induced force is determined as before and emerges as

$$
\begin{aligned}
F_{z}= & \left(\frac{I}{4 \pi \varepsilon_{0}^{2} c R^{4}}\right)\left(\left[\alpha_{\perp}^{2} \sin ^{2} \phi\left(1-3 \cos ^{2} \theta\right)+\alpha_{\|}^{2} \cos ^{2} \phi\right]\right. \\
& \times\left\{3 \hat{R}_{z} \cos k R \cos (\mathbf{k} \cdot \mathbf{R})+k R\left[3 \hat{R}_{z} \sin k R \cos (\mathbf{k} \cdot \mathbf{R})\right.\right. \\
& \left.+\hat{k}_{z} \cos k R \sin (\mathbf{k} \cdot \mathbf{R})\right]-k^{2} R^{2}\left[\hat{R}_{z} \cos k R \cos (\mathbf{k} \cdot \mathbf{R})\right. \\
& \left.\left.-\hat{k}_{z} \sin k R \sin (\mathbf{k} \cdot \mathbf{R})\right]\right\}-\left(\alpha_{\perp}^{2} \sin ^{2} \phi \sin ^{2} \theta+\alpha_{\|}^{2} \cos ^{2} \phi\right) \\
& \times\left\{k^{2} R^{2} \hat{R}_{z} \cos k R \cos (\mathbf{k} \cdot \mathbf{R})+k^{3} R^{3}\left[\hat{R}_{z} \sin k R \cos (\mathbf{k} \cdot \mathbf{R})\right.\right. \\
& \left.\left.\left.+\hat{k}_{z} \cos k R \sin (\mathbf{k} \cdot \mathbf{R})\right]\right\}\right)
\end{aligned}
$$

The leading term of Eq. (3.3), $F_{z}^{0}$, in the short-range region $(k R \ll 1)$, is found by taking the leading terms in the Taylor series expansions of $\sin k R, \cos k R, \sin (\mathbf{k} \cdot \mathbf{R})$, and $\cos (\mathbf{k} \cdot \mathbf{R})$ to give

$$
F_{z}^{0}=\left(\frac{3 I \hat{R}_{z}}{4 \pi \varepsilon_{0}^{2} c R^{4}}\right)\left[\alpha_{\perp}^{2} \sin ^{2} \phi\left(1-3 \cos ^{2} \theta\right)+\alpha_{\|}^{2} \cos ^{2} \phi\right] .
$$

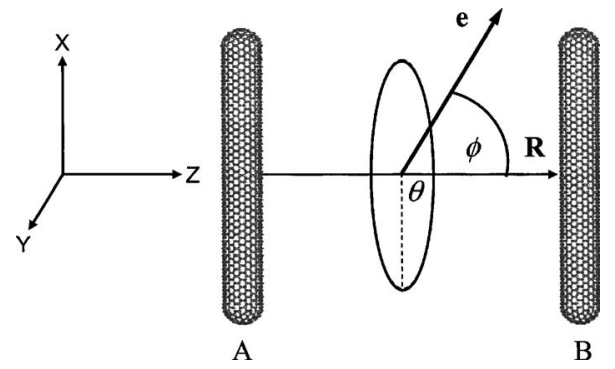

FIG. 6. Geometry of a system of parallel cylindrical particles irradiated in a fixed direction, here illustrated with nanotubes.

The case where the two nanoparticles have spherical symmetry can be represented by imposing the conditions that $\alpha_{\perp}$ and $\alpha_{\|}$are replaced by $\alpha \equiv 1 / 3 \alpha_{i i}$ and also $\theta=0$. In the short range the corresponding result is given by

$$
F_{z}^{0}=\left(\frac{3 \alpha^{2} I \hat{R}_{z}}{4 \pi \varepsilon_{0}^{2} c R^{4}}\right)\left(1-3 \sin ^{2} \phi\right)
$$

On isotropically averaging the system with respect to the incoming light, the force of Eq. (3.5) becomes solely attractive and is written as

$$
\left\langle F_{z}^{0}\right\rangle=-\frac{3 \alpha^{2} I \hat{R}_{z}}{4 \pi \varepsilon_{0}^{2} c R^{4}}
$$

an outcome whose unequivocal sign illustrates why the laserinduced force is often termed an optical binding force $[19,21]$.

Finally, and for completeness, the case of identical cylindrical particles with static dipole moments can be entertained, generating additional terms to the equation for the laser-induced force, Eq. (3.3). The second term of Eq. (2.6) is written as

$$
\begin{aligned}
\Delta E_{\text {ind }}= & \left(\frac{I}{2 \varepsilon_{0} c}\right) \operatorname{Re}\left(\sum_{J, K} \sin ^{2} \phi \cos ^{2} \theta \beta_{Z J Z}^{A} V_{J K}(0, \mathbf{R}) \mu_{K}^{B}\right. \\
& -\sin ^{2} \phi \sin \theta \cos \theta \beta_{Z J Y}^{A} V_{J K}(0, \mathbf{R}) \mu_{K}^{B} \\
& -\sin \phi \cos \phi \cos \theta \beta_{Z J X}^{A} V_{J K}(0, \mathbf{R}) \mu_{K}^{B}-\sin ^{2} \phi \sin \theta \\
& \times \cos \theta \beta_{Y J Z}^{A} V_{J K}(0, \mathbf{R}) \mu_{K}^{B} \\
& +\sin ^{2} \phi \sin ^{2} \theta \beta_{Y J Y}^{A} V_{J K}(0, \mathbf{R}) \mu_{K}^{B}+\sin \phi \cos \phi \\
& \times \sin \theta \beta_{Y J X}^{A} V_{J K}(0, \mathbf{R}) \mu_{K}^{B} \\
& -\sin \phi \cos \phi \cos \theta \beta_{X J Z}^{A} V_{J K}(0, \mathbf{R}) \mu_{K}^{B}+\sin \phi \\
& \times \cos \phi \sin \theta \beta_{X J Y}^{A} V_{J K}(0, \mathbf{R}) \mu_{K}^{B} \\
& \left.+\cos { }^{2} \phi \beta_{X J X}^{A} V_{J K}(0, \mathbf{R}) \mu_{K}^{B}+A \leftrightarrow B\right)
\end{aligned}
$$

where $A \leftrightarrow B$ denotes the interchange of particles $A$ and $B$. Next we identify that, for each cylindrical particle, the static dipole moment (to be designated $\mu_{\|}$) points in the $X$ 
direction; also only $\beta_{X X X}=\beta_{\|}, \beta_{Y Y X}=\beta_{Z Z X}=\beta_{\perp_{1}}, \beta_{Y X Y}$ $=\beta_{Z X Z}=\beta_{\perp_{2}}$, and $\beta_{X Y Y}=\beta_{X Z Z}=\beta_{\perp_{3}}$ are nonzero. Thus, with the explicit form of the $V_{J K}$, Eq. (3.7) gives

$$
\Delta E_{\mathrm{ind}}=\left(\frac{I \mu_{\|}}{4 \pi \varepsilon_{0}^{2} c R^{3}}\right)\left(\beta_{\perp_{2}} \sin ^{2} \phi+\beta_{\|} \cos ^{2} \phi\right) .
$$

Again, the superscripts $A$ and $B$ can be omitted at this stage. Thus, the complete expression for the polar cylindrical pair is given by the sum of this result and Eq. (3.3). In the shortrange region, this is determined as

$$
\begin{aligned}
F_{z}^{0}= & \left(\frac{3 I \hat{R}_{z}}{4 \pi \varepsilon_{0}^{2} c R^{4}}\right)\left[\alpha_{\perp}^{2} \sin ^{2} \phi\left(1-3 \cos ^{2} \theta\right)+\alpha_{\|}^{2} \cos ^{2} \phi\right. \\
& \left.+\mu_{\|}\left(\beta_{\perp_{2}} \sin ^{2} \phi+\beta_{\|} \cos ^{2} \phi\right)\right] .
\end{aligned}
$$

Although, as observed earlier, the force represented by Eq. (3.9) will necessarily represent only a correction to a substantially larger dipole-dipole force, it is in principle measurable through its intensity dependence.

\section{B. Tumbling pair}

A second case of interest concerning a pair of cylindrical nanoparticles is where the pair freely tumbles in the field of the input radiation, the two particles having arbitrary mutual orientation. Here the angle between the long axis of each particle and the $\mathbf{R}$ vector (the $Z$ direction of the laboratory frame) is defined as $\phi_{\xi}$-see Fig. 7(a). Furthermore, $\theta_{A B}$ is the angle between the long-axis projections of particles $A$ and $B$ on the plane perpendicular to $\mathbf{R}$, and the $X$ direction of the system frame is chosen such that the long axis of particle $A$ resides in the $X Z$ plane-see Fig. 7(b). To secure a result for the cylindrical pair that is isotropically averaged with respect to the incoming light, a phased-average method [17] is required to account for the phase factor $\exp (i \mathbf{k} \cdot \mathbf{R})$ in Eq. (2.6) - a feature that reflects the creation and annihilation of

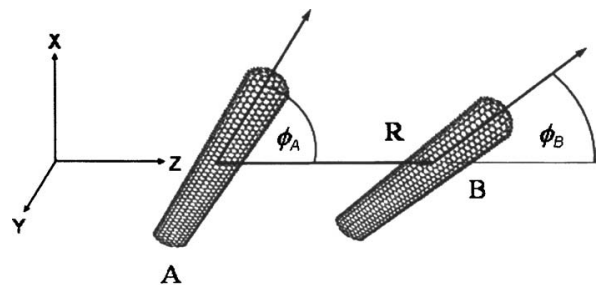

(a)

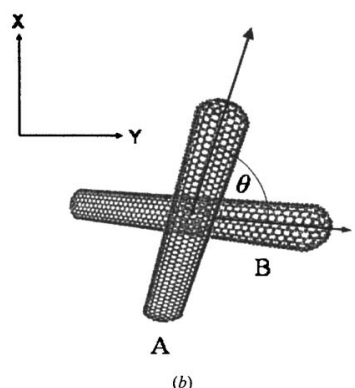

FIG. 7. Geometry of a pair of particles with fixed, arbitrary mutual orientation.

real photons at differing positions, i.e., one at $A$ and the other at $B$. Hence, with nonpolar particles, we obtain from the first term of Eq. (2.6)

$$
\begin{aligned}
\Delta E_{\text {ind }}= & \left(\frac{I}{\varepsilon_{0} c}\right) \operatorname{Re}\left[\frac{1}{3} j_{0}(k R) \alpha_{I J}^{A} V_{J K} \alpha_{K I}^{B}-\frac{3}{2} j_{2}(k R)\right. \\
& \left.\times\left(-\frac{1}{3} \hat{R}_{I} \alpha_{I J}^{A} V_{J K} \alpha_{K L}^{B} \hat{R}_{L}+\frac{1}{9} \alpha_{I J}^{A} V_{J K} \alpha_{K I}^{B}\right)\right],
\end{aligned}
$$

using the identities $e_{i} \bar{e}_{l}=1$ and $e_{i} \hat{k}_{i}=0$. Here, $j_{n}(k R)$ are spherical Bessel function,

$$
j_{0}(k R)=\frac{\sin k R}{k R}, \quad j_{2}(k R)=\left(\frac{-1}{k R}+\frac{3}{k^{3} R^{3}}\right) \sin k R-\frac{3 \cos k R}{k^{2} R^{2}} .
$$

From Eq. (3.10), by contracting $\alpha_{I J}^{A}$ and $\alpha_{K I}^{B}$ with the explicit form of the $V_{J K}$ tensor and defining the resulting equation in terms of $\alpha_{\perp}$ and $\alpha_{\|}$-achieved by referring the polarizability to the laboratory frame - we arrive at

$$
\begin{aligned}
\Delta E_{\text {ind }}= & \left(\frac{I k^{3}}{8 \pi \varepsilon_{0}^{2} c}\right)\left[\left\{\left(1-\eta \cos ^{2} \phi_{A}\right)\left[\alpha_{\|}^{2} \cos ^{2} \theta_{A B}\left(1-\eta \cos ^{2} \phi_{B}\right)+\alpha_{\|} \alpha_{\perp} \sin ^{2} \theta_{A B}\right]+\alpha_{\|} \alpha_{\perp} \sin ^{2} \theta_{A B}\left(1-\eta \cos ^{2} \phi_{B}\right)+\alpha_{\perp}^{2} \cos ^{2} \theta_{A B}\right.\right. \\
& \left.-\alpha_{\|}^{2} \eta^{2} \cos \theta_{A B} \sin \phi_{A} \sin \phi_{B} \cos \phi_{A} \cos \phi_{B}-2 \alpha_{\|}^{2}\left(1-\eta \sin ^{2} \phi_{A}\right)\left(1-\eta \sin ^{2} \phi_{B}\right)\right\} \\
& \times\left(\frac{\sin ^{2} k R}{k^{3} R^{3}}+\frac{2 \sin k R \cos k R}{k^{4} R^{4}}+\frac{\cos ^{2} k R}{k^{5} R^{5}}-\frac{\sin ^{2} k R}{k^{5} R^{5}}-\frac{\sin k R \cos k R}{k^{6} R^{6}}\right)
\end{aligned}
$$




$$
\begin{aligned}
& -\left\{\left(1-\eta \cos ^{2} \phi_{A}\right)\left[\alpha_{\|}^{2} \cos ^{2} \theta_{A B}\left(1-\eta \cos ^{2} \phi_{B}\right)+\alpha_{\|} \alpha_{\perp} \sin ^{2} \theta_{A B}\right]+\alpha_{\|} \alpha_{\perp} \sin ^{2} \theta_{A B}\left(1-\eta \cos ^{2} \phi_{B}\right)\right. \\
& \left.+\alpha_{\perp}^{2} \cos ^{2} \theta_{A B}-\alpha_{\|}^{2} \eta^{2} \cos \theta_{A B} \sin \phi_{A} \sin \phi_{B} \cos \phi_{A} \cos \phi_{B}\right\}\left(\frac{\sin k R \cos k R}{k^{2} R^{2}}+\frac{\cos ^{2} k R}{k^{3} R^{3}}-\frac{\sin k R \cos k R}{k^{4} R^{4}}\right) \\
& +\left[\alpha_{\|}^{2} \eta^{2} \cos \theta_{A B} \sin \phi_{A} \sin \phi_{B} \cos \phi_{A} \cos \phi_{B}-2 \alpha_{\|}^{2}\left(1-\eta \sin ^{2} \phi_{A}\right)\left(1-\eta \sin ^{2} \phi_{B}\right)\right] \\
& \times\left(\frac{3 \sin ^{2} k R}{k^{5} R^{5}}-\frac{\sin ^{2} k R}{k^{3} R^{3}}-\frac{3 \cos ^{2} k R}{k^{5} R^{5}}+\frac{3 \sin k R \cos k R}{k^{6} R^{6}}-\frac{4 \sin k R \cos k R}{k^{4} R^{4}}\right) \\
& \left.-\alpha_{\|}^{2} \eta^{2} \cos \theta_{A B} \sin \phi_{A} \sin \phi_{B} \cos \phi_{A} \cos \phi_{B}\left(-\frac{\sin k R \cos k R}{k^{2} R^{2}}-\frac{3 \cos ^{2} k R}{k^{3} R^{3}}+\frac{3 \sin k R \cos k R}{k^{4} R^{4}}\right)\right],
\end{aligned}
$$

where $\eta=\left(\alpha_{\|}-\alpha_{\perp}\right) / \alpha_{\|}$. The laser-induced force of the tumbling pair system is then

$$
\begin{aligned}
F_{z}= & \left(\frac{I k^{3} \hat{R}_{z}}{8 \pi \varepsilon_{0}^{2} c}\right)\left(\frac { \operatorname { c o s } ^ { 2 } k R - \operatorname { s i n } ^ { 2 } k R } { k R ^ { 2 } } \left\{\left(1-\eta \cos ^{2} \phi_{A}\right)\left[\alpha_{\|}^{2} \cos ^{2} \theta_{A B}\left(1-\eta \cos ^{2} \phi_{B}\right)+\alpha_{\|} \alpha_{\perp} \sin ^{2} \theta_{A B}\right]+\alpha_{\|} \alpha_{\perp} \sin ^{2} \theta_{A B}\left(1-\eta \cos ^{2} \phi_{B}\right)\right.\right. \\
& \left.+\alpha_{\perp}^{2} \cos ^{2} \theta_{A B}\right\}-\frac{\sin k R \cos k R}{k^{2} R^{3}}\left(6 \left\{\left(1-\eta \cos ^{2} \phi_{A}\right)\left[\alpha_{\|}^{2} \cos ^{2} \theta_{A B}\left(1-\eta \cos ^{2} \phi_{B}\right)+\alpha_{\|} \alpha_{\perp} \sin ^{2} \theta_{A B}\right]+\alpha_{\|} \alpha_{\perp} \sin ^{2} \theta_{A B}\right.\right. \\
& \left.\left.\times\left(1-\eta \cos ^{2} \phi_{B}\right)+\alpha_{\perp}^{2} \cos ^{2} \theta_{A B}\right\}-8 \alpha_{\|}^{2} \eta^{2} \cos \theta_{A B} \sin \phi_{A} \sin \phi_{B} \cos \phi_{A} \cos \phi_{B}\right) \\
& +\frac{\sin ^{2} k R-\cos ^{2} k R}{k^{3} R^{4}}\left(6 \left\{\left(1-\eta \cos ^{2} \phi_{A}\right)\left[\alpha_{\|}^{2} \cos ^{2} \theta_{A B}\left(1-\eta \cos ^{2} \phi_{B}\right)+\alpha_{\|} \alpha_{\perp} \sin ^{2} \theta_{A B}\right]\right.\right. \\
& \left.+\alpha_{\|} \alpha_{\perp} \sin ^{2} \theta_{A B}\left(1-\eta \cos ^{2} \phi_{B}\right)+\alpha_{\perp}^{2} \cos ^{2} \theta_{A B}\right\}-14 \alpha_{\|}^{2} \eta^{2} \cos \theta_{A B} \sin \phi_{A} \sin \phi_{B} \cos \phi_{A} \cos \phi_{B} \\
& \left.+4 \alpha_{\|}^{2}\left(1-\eta \sin ^{2} \phi_{A}\right)\left(1-\eta \sin ^{2} \phi_{B}\right)\right)+\frac{\sin k R \cos k R}{k^{4} R^{5}}\left[1 6 \left\{( 1 - \eta \operatorname { c o s } ^ { 2 } \phi _ { A } ) \left[\alpha_{\|}^{2} \cos ^{2} \theta_{A B}\left(1-\eta \cos ^{2} \phi_{B}\right)\right.\right.\right. \\
& \left.\left.+\alpha_{\|} \alpha_{\perp} \sin ^{2} \theta_{A B}\right]+\alpha_{\|} \alpha_{\perp} \sin ^{2} \theta_{A B}\left(1-\eta \cos ^{2} \phi_{B}\right)+\alpha_{\perp}^{2} \cos ^{2} \theta_{A B}\right\} \\
& \left.+48 \alpha_{\|}^{2} \eta^{2} \cos _{A B} \sin \phi_{A} \sin _{B} \cos \phi_{A} \cos _{B}+32 \alpha_{\|}^{2}\left(1-\eta \sin ^{2} \phi_{A}\right)\left(1-\eta \sin ^{2} \phi_{B}\right)\right] \\
& +\left(\frac{\cos ^{2} k R-\sin ^{2} k R}{k^{5} R^{6}}-\frac{\sin _{k R} \cos _{k}}{k^{6} R^{7}}\right)\left(6 \left\{\left(1-\eta \cos ^{2} \phi_{A}\right)\left[\alpha_{\|}^{2} \cos ^{2} \theta_{A B}\left(1-\eta \cos ^{2} \phi_{B}\right)+\alpha_{\|} \alpha_{\perp} \sin ^{2} \theta_{A B}\right]\right.\right. \\
& \left.+\alpha_{\|} \alpha_{\perp} \sin ^{2} \theta_{A B}\left(1-\eta \cos ^{2} \phi_{B}\right)+\alpha_{\perp}^{2} \cos ^{2} \theta_{A B}\right\}-24 \alpha_{\|}^{2} \eta^{2} \cos \theta_{A B} \sin \phi_{A} \sin \phi_{B} \cos _{A} \cos \phi_{B} \\
& \left.\left.+24 \alpha_{\|}^{2}\left(1-\eta \sin ^{2} \phi_{A}\right)\left(1-\eta \sin ^{2} \phi_{B}\right)\right)\right) .
\end{aligned}
$$

The short-range asymptote $F_{z}^{0}$ is found by taking the leading terms in the Taylor series expansions of $\sin k R$ and $\cos k R$, giving

$$
\begin{aligned}
F_{z}^{0}= & \left(\frac{I \hat{R}_{z}}{8 \pi \varepsilon_{0}^{2} c R^{4}}\right)\left(4\left\{\left(1-\eta \cos ^{2} \phi_{A}\right)\left[\alpha_{\|}^{2} \cos ^{2} \theta_{A B}\left(1-\eta \cos ^{2} \phi_{B}\right)+\alpha_{\|} \alpha_{\perp} \sin ^{2} \theta_{A B}\right]+\alpha_{\|} \alpha_{\perp} \sin ^{2} \theta_{A B}\left(1-\eta \cos ^{2} \phi_{B}\right)+\alpha_{\perp}^{2} \cos ^{2} \theta_{A B}\right\}\right. \\
& \left.-10 \alpha_{\|}^{2} \eta^{2} \cos \theta_{A B} \sin \phi_{A} \sin \phi_{B} \cos \phi_{A} \cos \phi_{B}+4 \alpha_{\|}^{2}\left(1-\eta \sin ^{2} \phi_{A}\right)\left(1-\eta \sin ^{2} \phi_{B}\right)\right)
\end{aligned}
$$

In the tumbling-pair system under consideration, it is of further interest to determine an expression for the torque $\tau$ between the two cylindrical particles with respect to $\theta_{A B}$, as given by $\boldsymbol{\tau}=\left(-\partial \Delta E_{\text {ind }} / \partial \theta_{A B}\right) \hat{\mathbf{R}}$. Specifically, with $\phi_{\xi}=90^{\circ}$,

$$
\begin{aligned}
\boldsymbol{\tau}= & \left(\frac{I k^{3} \hat{\mathbf{R}}}{8 \pi \varepsilon_{0}^{2} c}\right)\left[\left(-\frac{\sin k R \cos k R}{k^{2} R^{2}}+\frac{\sin ^{2} k R-\cos ^{2} k R}{k^{3} R^{3}}\right) \alpha_{\|}^{2} \eta^{2} \sin 2 \theta_{A B}+\frac{\sin k R \cos k R}{k^{4} R^{4}}\left(3 \alpha_{\|}^{2} \eta^{2} \sin 2 \theta_{A B}-4 \alpha_{\perp}^{2}\right)+\left(\frac{\cos ^{2} k R-\sin ^{2} k R}{k^{5} R^{5}}\right.\right. \\
& \left.\left.-\frac{\sin k R \cos k R}{k^{6} R^{6}}\right)\left(\alpha_{\|}^{2} \eta^{2} \sin 2 \theta_{A B}-4 \alpha_{\perp}^{2}\right)\right] .
\end{aligned}
$$

In the short-range region the result $\tau^{0}$, signifying the leading term in the expansion of Eq. (3.15) emerges in the following explicit form: 


$$
\boldsymbol{\tau}^{0}=\left(\frac{I \hat{\mathbf{R}}}{8 \pi \varepsilon_{0}^{2} c R^{3}}\right)\left(\alpha_{\|}-\alpha_{\perp}\right)^{2} \sin 2 \theta_{A B} .
$$

This result is consistent with an energetically optimized orthogonal orientation of the particles' long axes for $\alpha_{\|} \neq \alpha_{\perp}$, i.e., $\tau^{0}$ is positive for angles $\theta_{A B}$ between $0^{\circ}$ and $90^{\circ}, 180^{\circ}$ and $270^{\circ}$, and negative for $90^{\circ}-180^{\circ}, 270^{\circ}-360^{\circ}$, the sign, physically, denoting repulsion and attraction between the pair, respectively. The result (3.16) also satisfies the necessary condition $\tau^{0}$ $=0$ for spherical particles, i.e., where $\alpha_{\|}=\alpha_{\perp}=\alpha$.

For completeness a pair of cylindrical particles with static dipole moments is now accommodated by deriving the appropriate additional terms to Eq. (3.13). Again, isotropic averaging is implemented but, in this case, the phase-averaged method is not required as both creation and annihilation of real photons occur at either particle $A$ or $B$. Therefore, using a standard averaging method on the second term of Eq. (2.6) we find

$$
\Delta E_{\text {ind }}=\left(\frac{I}{6 \varepsilon_{0} c}\right) \operatorname{Re}\left\{V_{J K}(0, \mathbf{R})\left[\beta_{I J I}^{\mathrm{A}}(k) \mu_{K}^{\mathrm{B}}+\beta_{I J I}^{\mathrm{B}}(k) \mu_{K}^{\mathrm{A}}\right]\right\} .
$$

By contracting the hyperpolarizabilities and the static dipole moments with the explicit form of $V_{J K}$ and casting the result in terms of $\beta_{\|}, \beta_{\perp_{1}}, \beta_{\perp_{2}}$, and $\beta_{\perp_{3}}$, Eq. (3.17) gives

$$
\begin{aligned}
\Delta E_{\mathrm{ind}}= & \left(\frac{I \mu_{\|}}{24 \pi \varepsilon_{0}^{2} c R^{3}}\right)\left\{\left(\beta_{\|}+2 \beta_{\perp_{2}}\right)\left(\cos \theta_{A B} \sin \phi_{A} \sin \phi_{B}-2 \cos \phi_{A} \cos \phi_{B}\right)+\left(\beta_{\perp_{1}}+\beta_{\perp_{2}}+\beta_{\perp_{3}}\right)\right. \\
& \times\left[\cos \theta_{A B} \sin \phi_{A} \sin \phi_{B}\left(\sin ^{2} \theta_{A B}+\cos ^{2} \phi_{B}\right)-2 \sin ^{2} \phi_{B} \cos \phi_{A} \cos \phi_{B}\right]+\left(\beta_{\|}-\beta_{\perp_{1}}-\beta_{\perp_{3}}\right)\left(\cos \theta_{A B} \sin \phi_{A} \sin \phi_{B} \cos { }^{2} \phi_{B}\right. \\
& \left.-2 \sin ^{2} \phi_{B} \cos \phi_{A} \cos \phi_{B}\right)+\left(\beta_{\|} \sin ^{2} \phi_{B}-\beta_{\perp_{1}}-\beta_{\perp_{3}}\right) \sin ^{2} \theta_{A B} \cos \theta_{A B} \sin \phi_{A} \sin \phi_{B}+\beta_{\perp_{2}}\left[\operatorname { c o s } \theta _ { A B } \operatorname { s i n } \phi _ { A } \operatorname { s i n } \phi _ { B } \left(\cos ^{2} \theta_{A B}\right.\right. \\
& \left.\left.\left.+\sin ^{2} \phi_{B}\right)-2 \cos \phi_{A}\left(\cos ^{3} \phi_{B}+\cos \phi_{B}\right)\right]+\beta_{\|}\left(\cos ^{3} \theta_{A B} \sin \phi_{A} \sin ^{3} \phi_{B}-2 \cos \phi_{A} \cos ^{3} \phi_{B}\right)\right\}
\end{aligned}
$$

The complete result for the laser-induced force in the described system, a polar particle pair, is found by summing the distance derivatives of Eqs. (3.18) and (3.13). In the short-range region, the result is

$$
\begin{aligned}
F_{z}^{0}= & \left(\frac{I \hat{R}_{z}}{8 \pi \varepsilon_{0}^{2} c R^{4}}\right)\left(4\left\{\left(1-\eta \cos ^{2} \phi_{A}\right)\left[\alpha_{\|}^{2} \cos ^{2} \theta_{A B}\left(1-\eta \cos ^{2} \phi_{B}\right)+\alpha_{\|} \alpha_{\perp} \sin ^{2} \theta_{A B}\right]+\alpha_{\|} \alpha_{\perp} \sin ^{2} \theta_{A B}\left(1-\eta \cos ^{2} \phi_{B}\right)+\alpha_{\perp}^{2} \cos ^{2} \theta_{A B}\right\}\right. \\
& -10 \alpha_{\|}^{2} \eta^{2} \sin \phi_{A} \sin \phi_{B} \cos \phi_{A} \cos \phi_{B} \cos \theta_{A B}+4 \alpha_{\|}^{2}\left(1-\eta \sin ^{2} \phi_{A}\right)\left(1-\eta \sin ^{2} \phi_{B}\right)+\mu_{\|}\left\{( \beta _ { \| } + 2 \beta _ { \perp _ { 2 } } ) \left(\cos \theta_{A B} \sin \phi_{A} \sin \phi_{B}\right.\right. \\
& \left.-2 \cos \phi_{A} \cos \phi_{B}\right)+\left(\beta_{\perp_{1}}+\beta_{\perp_{2}}+\beta_{\perp_{3}}\right)\left[\cos \theta_{A B} \sin \phi_{A} \sin \phi_{B}\left(\sin ^{2} \theta_{A B}+\cos ^{2} \phi_{B}\right)-2 \sin ^{2} \phi_{B} \cos \phi_{A} \cos \phi_{B}\right]+\left(\beta_{\|}-\beta_{\perp_{1}}\right. \\
& \left.-\beta_{\perp_{3}}\right)\left(\cos \theta_{A B} \sin \phi_{A} \sin \phi_{B} \cos ^{2} \phi_{B}-2 \sin ^{2} \phi_{B} \cos \phi_{A} \cos \phi_{B}\right)+\left(\beta_{\|} \sin ^{2} \phi_{B}-\beta_{\perp_{1}}-\beta_{\perp_{3}}\right) \sin ^{2} \theta_{A B} \cos \theta_{A B} \sin \phi_{A} \sin \phi_{B} \\
& +\beta_{\perp_{2}}\left[\cos \theta_{A B} \sin \phi_{A} \sin \phi_{B}\left(\cos ^{2} \theta_{A B}+\sin ^{2} \phi_{B}\right)-2 \cos \phi_{A}\left(\cos ^{3} \phi_{B}+\cos \phi_{B}\right)\right]+\beta_{\|}\left(\cos ^{3} \theta_{A B} \sin \phi_{A} \sin \phi_{B} \phi_{B}\right. \\
& \left.\left.\left.-2 \cos \phi_{A} \cos ^{3} \phi_{B}\right)\right\}\right) .
\end{aligned}
$$

\section{PARTICLES IN AN OPTICAL VORTEX}

\section{A. General torque expression}

It is well known that circularly polarized light comprises photons with spin angular momentum, and the equations derived in previous sections for optically induced forces are sufficiently general to admit this possibility. At this juncture, it appears that there is nothing particularly notable in the corresponding results that would justify the specific experimental deployment of circular polarization. Recently, however, optical beams with a distinctive type of angular momentum have become the subject of considerable interest. These optically engineered twisted beams (optical vortices) are endowed with what has become known as orbital angular momentum $[41,42]$. Here, the wave-front surface of the electromagnetic fields assumes helical form, while the intensity profile is typically annular with zero intensity at the beam center (where the phase is undefined). The most widely studied case of a twisted optical beam comprises LaguerreGaussian modes characterized by two integers $l$ and $p$. Here the former number $l$ is known as the winding number or topological charge and signifies that each photon carries orbital angular momentum of magnitude $l \hbar$, while $(p+1)$ denotes the number of radial nodes. With the appropriate quantum field representation now established [31,43], it is possible to formulate the involvement of twisted beams in laser-induced interparticle forces, and to identify some distinctive features that emerge.

Figure 8 illustrates two nanoparticles $A$ and $B$ optically trapped in a LG beam with $p=0$, i.e., an optical vortex with one radial node at the beam center. Both particles are equidistant from the beam center, and attention focuses on their 


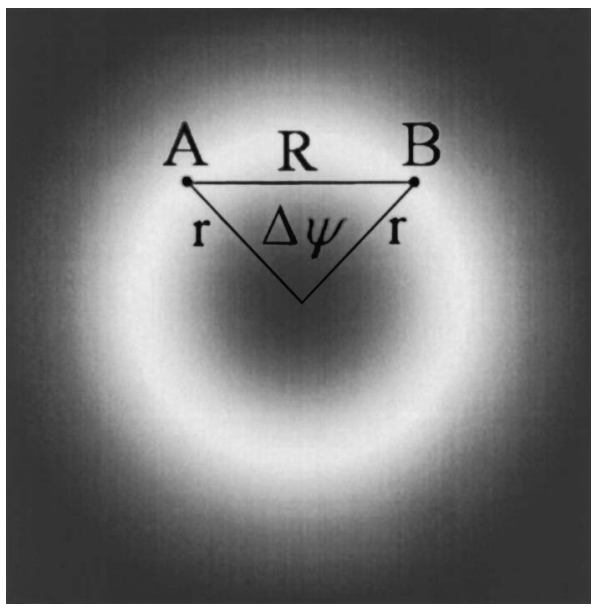

FIG. 8. Geometry of nanoparticle pair in a Laguerre-Gaussian beam.

laser-induced interaction. Under these conditions, it is interesting to determine an expression for the torque between the species, with respect to the beam axis $\hat{\zeta}(-Y$ in the system frame; cf. Sec. III B in which $\boldsymbol{\tau}$ is defined with respect to a different angle). Once again the starting point is the determination of $\Delta E_{\text {ind }}$, expedited by defining a system with the positions of $A$ and $B$ set in cylindrical coordinates. For simplicity, both particles are confined to a particular $\zeta$ valuethe distance of the particles from the beam center, $r$, is also fixed, while the azimuthal displacement angle $\Delta \psi=\psi_{B}-\psi_{A}$ is variable. The fourth-order perturbation method of Sec. II is again applied to the system with the aid of Figs. 2 and 3, now interpreting the input and output real photons as LG modes. The latter feature requires that the mode expansion of the field operator $\mathbf{d}^{\perp}\left(\mathbf{R}_{\xi}\right)$ within $H_{\text {int }}$ is modified, from Eq. (2.3), for $\mathrm{LG}$ photons and is accordingly redefined as [31]

$$
\begin{aligned}
\mathbf{d}^{\perp}(r)= & i \sum_{k, \lambda, l, p}\left(\frac{\hbar c k \varepsilon_{0}}{2 A_{l p} V}\right)^{1 / 2}\left\{\boldsymbol{\varepsilon}^{(\lambda)}(k \hat{\zeta}) a_{l p}^{(\lambda)}(k \hat{\zeta}) f_{l p}(r) \exp [i(k \zeta\right. \\
& \left.-l \psi)]-\overline{\boldsymbol{\varepsilon}}^{(\lambda)}(k \hat{\zeta}) a_{l p}^{\dagger(\lambda)}(k \hat{\zeta}) f_{l p}(r) \exp [-i(k \zeta-l \psi)]\right\} .
\end{aligned}
$$

Here $k \hat{\zeta}$ is the wave vector, $\boldsymbol{\varepsilon}^{(\lambda)}(k \hat{\zeta})$ is a polarization unit vector normal to $\hat{\zeta}\left[\overline{\boldsymbol{\varepsilon}}^{(\lambda)}(k \hat{\zeta})\right.$ being its complex conjugate], $a_{l p}^{\dagger(\lambda)}(k \hat{\zeta})$ and $a_{l p}^{(\lambda)}(k \hat{\zeta})$ denote creation and annihilation operators respectively (acting on a twisted-state mode $|n(k, \lambda, l, p)\rangle$ conventionally, i.e., as defined in Sec. II), and $A_{l p}$ are normalization constants. Additionally, the radial function $f_{l p}(r)$ is given by

$$
f_{l p}(r)=\frac{C_{p}^{|l|}}{\omega_{0}}\left[\frac{\sqrt{2} r}{\omega_{0}}\right]^{|l|} \exp \left(\frac{-r^{2}}{\omega_{0}^{2}}\right) L_{p}^{|l|}\left(\frac{2 r^{2}}{\omega_{0}^{2}}\right)
$$

where $C_{p}^{|l|}$ is a further normalization constant, $\omega_{0}$ defines the Gaussian beam waist at $z=0$, and $L_{p}^{|l|}$ is a generalized Laguerre polynomial.

Although the mode expansion (4.1) is taken over a complete set-one that is especially suited for throughput LG radiation-it is not well suited for virtual photons with variable directions of propagation, and its use in representing their interactions would lead to unnecessary complications. Therefore, for particle-virtual-photon coupling, the mode expansion (2.3) is retained at the appropriate positions in the second term of Eq. (2.4), while at the remaining positions the real photon coupling employs the LG mode expansion (4.1). Following a similar approach as in the Appendix, we arrive at

$$
\begin{aligned}
\Delta E_{\text {ind }}= & \left(\frac{I f_{l p}^{2}(r)}{\varepsilon_{0} c A_{l p}}\right) \operatorname{Re}\left(\varepsilon_{i}^{(\lambda)} \bar{\varepsilon}_{l}^{(\lambda)} \alpha_{i j}^{A}(k) V_{j k}(k, \mathbf{R}) \alpha_{k l}^{B}(k) \exp (l \Delta \psi)\right. \\
& \left.+\frac{1}{2} \varepsilon_{i}^{(\lambda)} \bar{\varepsilon}_{l}^{(\lambda)} V_{j k}(0, \mathbf{R})\left[\beta_{i j l}^{A}(k) \mu_{k}^{B}+\mu_{k}^{A} \beta_{i j l}^{B}(k)\right]\right)
\end{aligned}
$$

The inclusion of the phase factor $\exp (l \Delta \psi)$ in the first term of Eq. (4.3) is consistent with twisted beams, i.e., light that exhibits features associated with an orbital angular momentum $L$ of eigenvalue $l \hbar$. From Eq. (4.3), using $R=\sqrt{2} r(1$ $-\cos \Delta \psi)^{1 / 2}$ and for conciseness suppressing the $f_{l p}$ and $\alpha_{i j}$ dependence, the general expression for the laser-induced torque $\tau=\left(-\partial \Delta E_{\text {ind }} / \partial \Delta \psi\right) \hat{\mathbf{k}}$ emerges as

$$
\begin{aligned}
\boldsymbol{\tau}= & \left(\frac{I k^{3} f_{l p}^{2} \hat{\mathbf{k}}}{8 \pi \varepsilon_{0}^{2} c A_{l p}}\right)\left[2 \operatorname { R e } ( \varepsilon _ { i } ^ { ( \lambda ) } \overline { \varepsilon } _ { l } ^ { ( \lambda ) } \alpha _ { i j } ^ { A } \alpha _ { k l } ^ { B } ) \left[\frac{-\left(\delta_{j k}-\hat{R}_{j} \hat{R}_{k}\right) \sin \left(\sqrt{2} k r(1-\cos \Delta \psi)^{1 / 2}\right) \sin \Delta \psi}{2(1-\cos \Delta \psi)} \cos (l \Delta \psi)\right.\right. \\
& -\frac{\cos \left[\sqrt{2} k r(1-\cos \Delta \psi)^{1 / 2}\right]}{\sqrt{2} k r(1-\cos \Delta \psi)^{3 / 2}}\left[\left(\delta_{j k}-\hat{R}_{j} \hat{R}_{k}\right) l \sin (l \Delta \psi)(1-\cos \Delta \psi)+\left(\delta_{j k}-2 \hat{R}_{j} \hat{R}_{k}\right) \sin \Delta \psi \cos (l \Delta \psi)\right] \\
& +\left(\frac{\left(\delta_{j k}-3 \hat{R}_{j} \hat{R}_{k}\right) \sin \left(\sqrt{2} k r(1-\cos \Delta \psi)^{1 / 2}\right)}{2 k^{2} r^{2}(1-\cos \Delta \psi)^{2}}+\frac{\left(\delta_{j k}-3 \hat{R}_{j} \hat{R}_{k}\right) \cos \left[\sqrt{2} k r(1-\cos \Delta \psi)^{1 / 2}\right]}{2 \sqrt{2} k^{3} r^{3}(1-\cos \Delta \psi)^{5 / 2}}\right)(l \sin (l \Delta \psi)(1-\cos \Delta \psi) \\
& \left.\left.+\frac{3}{2} \cos (l \Delta \psi) \sin \Delta \psi\right)\right)+3 \operatorname{Re}\left[\varepsilon_{i}^{(\lambda)} \bar{\varepsilon}_{l}^{(\lambda)}\left(\beta_{i j l}^{A} \mu_{k}^{B}+\beta_{i j l}^{B} \mu_{k}^{A}\right)\right] \frac{\left(\delta_{j k}-3 \hat{R}_{j} \hat{R}_{k}\right) \sin \Delta \psi}{4 \sqrt{2} k^{3} r^{3}(1-\cos \Delta \psi)^{5 / 2}} .
\end{aligned}
$$




\section{B. Spherical nanoparticles}

A system that it is of particular interest, now to be examined in more detail, is where spherical nanoparticles are trapped in an annular intensity region as in Fig. 8. With an irradiating LG beam, the laser-induced energy shift for the system is determined by expressing the polarization unit vector in simplified cylindrical coordinates (again see Fig. 6). Defining the system in terms of the laboratory frame, with $\theta=0$ and $\boldsymbol{\varepsilon}=\sin \phi \mathbf{i}+\cos \phi \mathbf{k}$, and $\mu_{k}^{\xi}=0$ for spherical nanoparticles, Eq. (4.3) gives

$$
\begin{aligned}
\Delta E_{\text {ind }}= & \left(\frac{I f_{l p}^{2}}{2 \varepsilon_{0} c A_{l p}}\right) \operatorname{Re}\left(\sum_{J, K} \sin ^{2} \phi \alpha_{Z J}^{\mathrm{A}} V_{J K} \alpha_{K Z}^{\mathrm{B}}\right. \\
& -\sin \phi \cos \phi\left(\alpha_{Z J}^{\mathrm{A}} V_{J K} \alpha_{K X}^{\mathrm{B}}+\alpha_{X J}^{\mathrm{A}} V_{J K} \alpha_{K Z}^{\mathrm{B}}\right) \\
& \left.+\cos ^{2} \phi \alpha_{X J}^{\mathrm{A}} V_{J K} \alpha_{K X}^{\mathrm{B}}+A \leftrightarrow B\right) \cos (l \Delta \psi) .
\end{aligned}
$$

Taking the explicit form of the $V_{J K}$ tensor and recognizing that $\alpha_{X X}=\alpha_{Y Y}=\alpha_{Z Z}=\alpha$ for a spherical pair, Eq. (4.5) is rewritten as

$$
\begin{aligned}
\Delta E_{\text {ind }}= & \left(\frac{I f_{l p}^{2} \alpha^{2}}{4 \pi \varepsilon_{0}^{2} c A_{l p}}\right)\left[\left(1-3 \sin ^{2} \phi\right)\left(\frac{\cos k R}{R^{3}}+\frac{k \sin k R}{R^{2}}\right)\right. \\
& \left.-\frac{k^{2} \cos ^{2} \phi \cos k R}{R}\right] \cos (l \Delta \psi)
\end{aligned}
$$

leading to the following expression for the laser-induced torque:

$$
\begin{aligned}
\boldsymbol{\tau}= & \left(\frac{I k^{3} f_{l p}^{2} \alpha^{2} \hat{\mathbf{k}}}{4 \pi \varepsilon_{0}^{2} c A_{l p}}\right) \\
& \times\left[-\frac{\sin \left[\sqrt{2} k r(1-\cos \Delta \psi)^{1 / 2}\right]}{2(1-\cos \Delta \psi)} \cos ^{2} \phi \sin \Delta \psi \cos (l \Delta \psi)\right. \\
& -\frac{\cos \left[\sqrt{2} k r(1-\cos \Delta \psi)^{1 / 2}\right]}{2 \sqrt{2} k r(1-\cos \Delta \psi)^{3 / 2}}\left\{2 l \cos ^{2} \phi \sin (l \Delta \psi)(1\right. \\
& \left.-\cos \Delta \psi)+\left[\left(1-3 \sin ^{2} \phi\right)+\cos ^{2} \phi\right] \sin \Delta \psi \cos (l \Delta \psi)\right\} \\
& +\left(\frac{\left(1-3 \sin ^{2} \phi\right) \sin \left[\sqrt{2} k r\left(1-\cos ^{2} \psi\right]^{1 / 2}\right)}{2 k^{2} r^{2}(1-\cos \Delta \psi)^{2}}\right. \\
& \left.+\frac{\left(1-3 \sin ^{2} \phi\right) \cos \left[\sqrt{2} k r(1-\cos \Delta \psi)^{1 / 2}\right]}{2 \sqrt{2} k^{3} r^{3}(1-\cos \Delta \psi)^{5 / 2}}\right) \\
& \left.\times\left(l \sin (l \Delta \psi)(1-\cos \Delta \psi)+\frac{3}{2} \cos (l \Delta \psi) \sin \Delta \psi\right)\right]
\end{aligned}
$$

In the short-range region $(k r \ll 1)$ Eq. (4.7) becomes

$$
\begin{aligned}
\boldsymbol{\tau}^{0}= & \frac{I f_{l p}^{2} \alpha^{2}\left(1-3 \sin ^{2} \phi\right) \hat{\mathbf{k}}}{8 \sqrt{2} \pi \varepsilon_{0}^{2} r^{3} c A_{l p}(1-\cos \Delta \psi)^{5 / 2}}(l \sin (l \Delta \psi) \\
& \left.\times(1-\cos \Delta \psi)+\frac{3}{2} \cos (l \Delta \psi) \sin \Delta \psi\right) .
\end{aligned}
$$

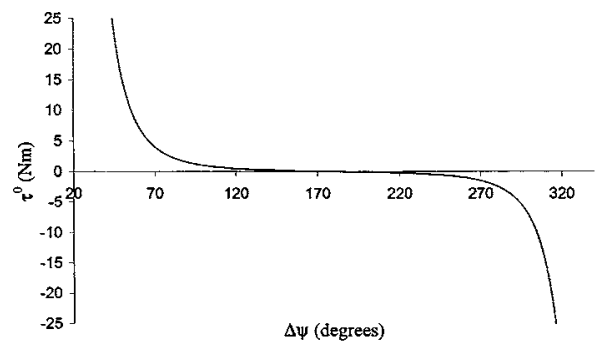

(a)

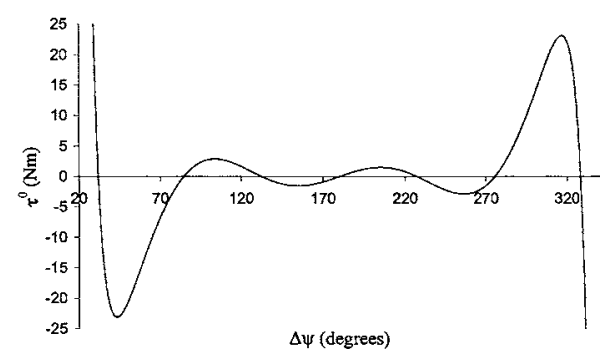

(b)

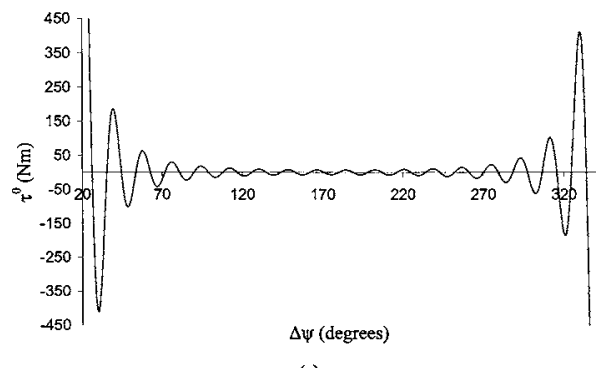

(c)

FIG. 9. Graphs of the magnitude of $\tau^{0}$ against $\Delta \psi$ for a nanoparticle pair in a LG beam with $l=$ (a) 0 , (b) 4 , and (c) 20 .

On inspection of Eqs. (4.7) and (4.8) it is apparent that, in comparison to conventional Gaussian laser light $(l=0$, for example) additional contributions to the interparticle torque will be observed with a LG beam $(l \neq 0)$. In the short-range region, and for three different values of $l$, graphs detailing the change in magnitude of $\tau^{0}$ with $\Delta \psi$ are exhibited in Figs. $9(\mathrm{a})-9(\mathrm{c})$. Although the rise of $\tau^{0}$ toward infinity as $\Delta \psi$ approaches $0^{\circ}$ (or equivalently $360^{\circ}$ ) is prominent on these diagrams, it is an artifice corresponding to the nonphysical case of particle overlap. Of greater interest is the physically significant observation that there are $(2 l-1)$ nodes, i.e., angular positions where $\tau^{0}=0$, of which it may be ascertained that $(l-1)$ correspond to maxima and $l$ to minima in $\Delta E_{\text {ind }}$. The latter energy minima signify values of $\Delta \psi$ for which the nanoparticle pair is mutually trapped-the detailed angular disposition of these is the subject of ongoing research. The above findings illustrate that the laser-induced mechanisms operative here differ significantly from those of established "optical spanner" methods_not least because, here, there is no involvement of any orbital angular momentum transfer from the laser beam. Further distinctions can be identified in comparison to dynamic holographic optical tweezer schemes [44], including the fact that trapped particles do not circulate around the annular high-intensity region of the twisted beam. 


\section{APPLICATIONS}

\section{A. Carbon nanotubes}

Recently, results have been secured for the van der Waals interactions between a pair of carbon nanotubes [45] - a material currently the subject of intense research due to its unique nanostructures and remarkable combination of conductive, steric, and mechanical properties [46]. Under suitable conditions the laser-induced interactions between nanotubes, as determined from the results in Sec. III, prove to be of significantly greater magnitude. With nanotubes of length $200 \mathrm{~nm}$ and radius $0.4 \mathrm{~nm}$, also setting $R=2 \mathrm{~nm}$ and $I=1$ $\times 10^{16} \mathrm{~W} \mathrm{~m}^{-2}$, values for the laser-induced force range from micronewtons to nanonewtons, depending on the nanotube orientations [15]. As these results exceed by many orders of magnitude the van der Waals forces at a similar distance, there are potentially important applications. Carbon nanotubes are excellent candidates for implementation as components in nanoelectromechanical switches [47,48], devices that have the potential to enable new technology in sensing, actuation, and computing. Operating at the nanometer scale, the electrostatic Coulombic forces associated with the operation of such devices are comparable with chemical binding forces, and since van der Waals interactions play an important role, laser-induced interactions may potentially prove to be another major influencing factor-but one that offers the possibility of experimental control. Other tubular particles for which the results of Sec. III could be applied are the increasing number of noncarbon nanotubes, including those composed of boron nitride $[49,50]$ and the theoretically proposed boron nanotubes $[51,52]$, which have only very recently been synthesized [53]. The natural light-harvesting systems in the chromosomes of the green bacteria Chloroflexus aurantiacus [54,55]—-systems that are composed of cylindrical aggregates consisting of a large number of bacteriochlorophyll molecules - and the artificial light harvesters of carbocyanine dye molecules [56] that self-assemble into tubular aggregates, may also prove amenable to the application of our results.

\section{B. Dielectric microparticles}

As developed above, the equations for optomechanical forces are cast in terms of dielectric propertiespolarizability and hyperpolarizability, etc. For particles of micrometer size where it is more appropriate to express results in terms of bulk susceptibilities, the necessary conversions are readily effected by use of the Lorentz local-field tensor [57]. Formally, such corrections arise within the QED formalism through (complex) refractive index modifications to the electromagnetic field expansions [58]. Developing these media corrections also permits laser-induced forces between particles in a host medium to be addressed-particles suspended in a liquid for example-without compromising the rigor of the QED development. Here, modifications to the character of the virtual photon coupling through a refractive and dissipative medium lead to a modified form of the retarded resonance coupling tensor-see Ref. [59] for details. It is noteworthy that, although the scale of laser-induced compression is very small in the microscopic systems de- fined earlier, in a solid that is sufficiently transparent to the laser pulse, this compression would be scaled up by the large number of particle pairs and could result in a detectable compression. Since such a force exists only while the laser pulse is present, application of a laser pulse train would produce a cycle of compression and expansion with a period equal to that of the laser pulse interval. However, it needs to be borne in mind that, at an irradiance of $1 \times 10^{16} \mathrm{~W} \mathrm{~m}^{-2}$, there will be a significant radiation pressure if the material is not $100 \%$ transparent. Such levels of intensity can produce a radiation pressure alone of about $50 \mathrm{~atm}$, depending on the surface reflectivity.

\section{Bose-Einstein condensates}

In theoretical studies, laser-induced interactions have already been used to engineer the mesoscopic properties of weakly interacting Bose-Einstein condensates (BECs). Usually the condensate is stabilized by the presence of a harmonic trap which counters the repulsive mean-field energyitself a composite of zero-point and short-range dispersion energies-but through a laser-induced interaction method it is possible for the BEC to be self-trapped. This is achieved by directing a triad of laser beams in such a way as to exclude the short-range $\left(R^{-3}\right)$ component of the dipole-dipole interaction-which does not allow for such self-binding. This in turn leads to the long-range $\left(R^{-1}\right)$ attractive potential balancing the repulsive mean-field energy and, hence, a harmonic trap is no longer required to support the condensate [26-30]. It is possible for the mean-field energy to be attractive in nature-for example, in BECs composed of ${ }^{7} \mathrm{Li}$ atoms - and, in this instance, the condensate undergoes a typical cycle of growth and collapse until a stationary state is reached [60]. For such BECs to be sustained the occupation number, i.e., the number of atoms in the condensate, must not exceed a limiting value; the latter is determined by the balance between the competing forces [61] and can be manipulated by a laser-induced force. Consequently a control of the maximum number of atoms in the condensate is possible-also, potentially, its rate of growth. Thus the prospect emerges of enhancing the dynamics of a coherent Raman photoassociation process (also known as superchemistry) exploiting its dependence on the number of particles in the product mode [62], and the possibility of control over the interparticle interactions [63]. Although this type of chemistry has not yet been experimentally observed, partial success has been reported in an experiment to create molecular condensates by photoassociation [64]. ${ }^{1}$ Molecular BEC systems are expected to be directly amenable to the laser-induced concepts discussed in this paper.

\section{Other applications}

In conclusion we observe that other areas of application may be identified for the principles that we have described. One is the possible application of laser-induced opto-

\footnotetext{
${ }^{1}$ Note, molecular condensates have been successfully observed by using Feshbash resonance techniques [65].
} 
TABLE I. All states and their associated energies associated with the states sequences exhibited in Fig. 2.

\begin{tabular}{ccc}
\hline \hline $\begin{array}{c}\text { System state } \\
\left|r_{k}^{m}\right\rangle\end{array}$ & Ket & Energy \\
$E_{r_{k}^{m}}$
\end{tabular}

mechanical forces between suspended particles in the transport of microfluids, a context associated with increasingly important techniques for mixing and pumping extremely small volumes in laboratory-on-a-chip and associated devices $[66,67]$. An application of potential high-resolution spectroscopic interest is the identification of intensitydependent molecular vibrational frequencies, a feature that may arise through laser-induced modifications to the interatomic force fields associated with vibrational modes. Work on this is currently under way.

\section{SUMMARY}

Under intense laser radiation, optically induced forces and torques between nanoparticles occur by pairwise processes of stimulated photon scattering. By a quantum electrodynamical analysis, it is possible to identify the quantized nature of the fundamental particle interactions with the throughput radiation, and also in the electromagnetic coupling between particles. The application of these methods in this paper has enabled determination of a general expression for the laser-induced force-incorporating not only terms associated with the dynamic form of coupling, but also a less well-known, static form. Additionally, detailed equations have been derived for nanoparticle systems of both cylindrical and spherical symmetries. The results clearly illustrate that pairwise optomechanical forces may be either positive or negative, according to conditions - and an explanation is given for why the latter, often termed "optical binding" forces, prevail in the case of randomly configured, spherical nanoparticles. It is further shown that, on replacing conventional laser light with a LG optical vortex beam, i.e., one endowed with orbital angular momentum, additional torque features arise. Together, such laser-induced interactions differ significantly from those involved in established optical tweezer methods. The prospect of optically influencing the forces and torques between nanoparticles suggest its future implementation in a variety of future applications.

\section{ACKNOWLEDGMENTS}

D.L.A. would like to thank Professor Miles Padgett for helpful discussions on LG beams. We also thank EPSRC for funding this work.

\section{APPENDIX}

To clarify the procedure by which Eq. (2.6) is derived from the second term of Eq. (2.4) it is helpful to utilize the state-sequence diagrams of Figs. 4 and 5-a calculational aid that has several key advantages over the more familiar timeordered diagrams of Figs. 2 and 3. In these illustrations the circles to the left and right of each box delineate particles $A$ and $B$, respectively, with an empty circle representing a ground state and a gray-filled circle a virtual state. Also, $p$ denotes the presence of a virtual photon of mode $(\mathbf{p}, \varepsilon)$ and the symbol $k$ symbolizes a real photon with wave vector $\mathbf{k}$. Each box in the state-sequence diagram is linked to a reference position $(m, k)$, where $m$ and $k$ are the vertex and step number, respectively, and a matter-photon interaction event is denoted by a connector between boxes. In a single statesequence diagram (either Fig. 4 or 5 ) each of the 24 quantum channels can be traced from the initial to the final state, and corresponds to one member of a complete set of 24 timeordered diagrams whose construction would otherwise be required. Taking Fig. 4 as an example, for which all states and their associated energies are given by Table I, the analysis of one specific quantum channel can be illustrated for the case $|i\rangle \rightarrow\left|r_{1}^{1}\right\rangle \rightarrow\left|r_{2}^{1}\right\rangle \rightarrow\left|r_{3}^{1}\right\rangle \rightarrow|i\rangle$, signified by the lowest pathway. The one-photon parts of $\Delta E$ [each bracket in the numerator of Eq. (2.4)] may be determined by inserting Eq. (2.2) and then Eq. (2.3) into the second term of Eq. (2.4) to yield

$$
\begin{aligned}
\left\langle r\left|H_{\mathrm{int}}\right| i\right\rangle= & \left\langle A_{\alpha}, B_{0} ; n-1,0\right|-\varepsilon_{0}^{-1}\left[\boldsymbol{\mu}(A) \cdot \mathbf{d}^{\perp}\left(\mathbf{R}_{A}\right)\right. \\
& \left.+\boldsymbol{\mu}(B) \cdot \mathbf{d}^{\perp}\left(\mathbf{R}_{B}\right)\right]\left|A_{0}, B_{0} ; n, 0\right\rangle=-\varepsilon_{0}^{-1}\left\langle A_{\alpha}|\boldsymbol{\mu}(A)| A_{0}\right\rangle \\
& \times\left\langle n-1(\mathbf{k}, \lambda)\left|\mathbf{d}^{\perp}\left(\mathbf{R}_{A}\right)\right| n(\mathbf{k}, \lambda)\right\rangle \\
= & -i\left(\frac{n \hbar c k}{2 \varepsilon_{0} V}\right)^{1 / 2} e_{i}^{(\lambda)} \mu_{i}^{\alpha 0} \exp \left[i\left(\mathbf{k} \cdot \mathbf{R}_{A}\right)\right], \\
\left\langle s\left|H_{\mathrm{int}}\right| r\right\rangle= & \left\langle A_{0}, B_{0} ; n-1,1\right|-\varepsilon_{0}^{-1}\left[\boldsymbol{\mu}(A) \cdot \mathbf{d}^{\perp}\left(\mathbf{R}_{A}\right)\right. \\
& \left.+\boldsymbol{\mu}(B) \cdot \mathbf{d}^{\perp}\left(\mathbf{R}_{B}\right)\right]\left|A_{\alpha}, B_{0} ; n-1,0\right\rangle \\
= & -\varepsilon_{0}^{-1}\left\langle A_{0}|\boldsymbol{\mu}(A)| A_{\alpha}\right\rangle\left\langle 1(\mathbf{p}, \varepsilon)\left|\mathbf{d}^{\perp}\left(\mathbf{R}_{A}\right)\right| 0(\mathbf{p}, \varepsilon)\right\rangle \\
= & i \sum_{\mathbf{p}, \varepsilon}\left(\frac{\hbar c p}{2 \varepsilon_{0} V}\right)^{1 / 2} \bar{e}_{j}^{(\varepsilon)} \mu_{j}^{0 \alpha} \exp \left[-i\left(\mathbf{p} \cdot \mathbf{R}_{A}\right)\right], \quad(\mathrm{A} 2) \\
\left\langle t\left|H_{\mathrm{int}}\right| s\right\rangle= & \left\langle A_{0}, B_{\beta} ; n-1,0\right|-\varepsilon_{0}^{-1}\left[\boldsymbol{\mu}(A) \cdot \mathbf{d}^{\perp}\left(\mathbf{R}_{A}\right)\right. \\
& \left.+\boldsymbol{\mu}(B) \cdot \mathbf{d}^{\perp}\left(\mathbf{R}_{B}\right)\right]\left|A_{0}, B_{0} ; n-1,1\right\rangle \\
= & -\varepsilon_{0}^{-1}\left\langle B_{\beta}|\boldsymbol{\mu}(B)| B_{0}\right\rangle\left\langle 0(\mathbf{p}, \varepsilon)\left|\mathbf{d}^{\perp}\left(\mathbf{R}_{B}\right)\right| 1(\mathbf{p}, \varepsilon)\right\rangle \\
= & -i \sum_{\mathbf{p}, \varepsilon}\left(\frac{\hbar c p}{2 \varepsilon_{0} V}\right)^{1 / 2} e_{k}^{(\varepsilon)} \mu_{k}^{\beta 0} \exp \left[i\left(\mathbf{p} \cdot \mathbf{R}_{B}\right)\right]
\end{aligned}
$$




$$
\begin{aligned}
\left\langle i\left|H_{\text {int }}\right| t\right\rangle= & \left\langle A_{0}, B_{0} ; n, 0\right|-\varepsilon_{0}^{-1}\left[\boldsymbol{\mu}(A) \cdot \mathbf{d}^{\perp}\left(\mathbf{R}_{A}\right)\right. \\
& \left.+\boldsymbol{\mu}(B) \cdot \mathbf{d}^{\perp}\left(\mathbf{R}_{B}\right)\right]\left|A_{0}, B_{\beta} ; n-1,0\right\rangle \\
= & -\varepsilon_{0}^{-1}\left\langle B_{0}|\boldsymbol{\mu}(B)| B_{\beta}\right\rangle\left\langle n(\mathbf{k}, \lambda)\left|\mathbf{d}^{\perp}\left(\mathbf{R}_{B}\right)\right| n-1(\mathbf{k}, \lambda)\right\rangle \\
= & i\left(\frac{n \hbar c k}{2 \varepsilon_{0} V}\right)^{1 / 2} \bar{e}_{l}^{(\lambda)} \mu_{l}^{0 \beta} \exp \left[-i\left(\mathbf{k} \cdot \mathbf{R}_{B}\right)\right],
\end{aligned}
$$

where $\boldsymbol{\mu}(\xi)$ and $\mathbf{d}^{\perp}\left(\mathbf{R}_{\xi}\right)$ operate on $\left|\mathrm{mat}_{n}\right\rangle$ and $\left|\operatorname{rad}_{n}\right\rangle$, respec- tively, using the mode expansions given in Sec. II. Also, summation over all virtual photon wave vectors $\mathbf{p}$ and polarizations $\varepsilon$ is necessarily entailed but the corresponding real photon variables, i.e., $\mathbf{k}$ and $\lambda$, are fixed to a single value. The denominator of Eq. (2.4), in this case, is $\left[\left(E_{A}^{\alpha 0}-\hbar c k\right)\right.$ $\left.\times(\hbar c k-\hbar c p)\left(E_{B}^{\beta 0}-\hbar c k\right)\right]^{-1}$ where $E_{A}^{\alpha 0}$ is shorthand for $E_{A}^{\alpha}$ $-E_{A}^{0}$, for example. By repeating this process for the other 23 quantum channels, summing all the results, and simplifying, the second term of Eq. (2.4) becomes

$$
\begin{aligned}
\Delta E= & \left(\frac{n \hbar c k}{2 \varepsilon_{0} V}\right) \operatorname{Re}\left\{e _ { i } ^ { ( \lambda ) } \overline { e } _ { l } ^ { ( \lambda ) } \operatorname { e x p } [ - \mathrm { i } ( \mathbf { k } \cdot \mathbf { R } ) ] [ \sum _ { \mathbf { p } , \varepsilon } \frac { p } { 2 \varepsilon _ { 0 } } ( \frac { \overline { e } _ { j } ^ { ( \varepsilon ) } e _ { k } ^ { ( \varepsilon ) } \operatorname { e x p } [ i ( \mathbf { p } \cdot \mathbf { R } ) ] } { k - p } + \frac { \overline { e } _ { k } ^ { ( \varepsilon ) } e _ { j } ^ { ( \varepsilon ) } \operatorname { e x p } [ - i ( \mathbf { p } \cdot \mathbf { R } ) ] } { - k - p } ) ] \left[\frac{\mu_{i}^{0 \alpha} \mu_{j}^{\alpha 0} \mu_{k}^{0 \beta} \mu_{l}^{\beta 0}}{\left(E_{A}^{\alpha 0}-\hbar c k\right)\left(E_{B}^{\beta 0}-\hbar c k\right)}\right.\right. \\
& \left.\left.+\frac{\mu_{j}^{0 \alpha} \mu_{i}^{\alpha 0} \mu_{k}^{0 \beta} \mu_{l}^{\beta 0}}{\left(E_{A}^{\alpha 0}+\hbar c k\right)\left(E_{B}^{\beta 0}-\hbar c k\right)}+\frac{\mu_{i}^{0 \alpha} \mu_{j}^{\alpha 0} \mu_{l}^{0 \beta} \mu_{k}^{\beta 0}}{\left(E_{A}^{\alpha 0}-\hbar c k\right)\left(E_{B}^{\beta 0}+\hbar c k\right)}+\frac{\mu_{j}^{0 \alpha} \mu_{i}^{\alpha 0} \mu_{l}^{0 \beta} \mu_{k}^{\beta 0}}{\left(E_{A}^{\alpha 0}+\hbar c k\right)\left(E_{B}^{\beta 0}+\hbar c k\right)}\right]\right\},
\end{aligned}
$$

with the identities $\mu_{i}^{0 \alpha}=-\mu_{i}^{\alpha 0}$, etc. The first expression in square brackets of Eq. (A5) is resolved [37] to give $V_{i j}(k, \mathbf{R})$ of Eq. (2.7), and the second is the product of $-\alpha$ with itself; the explicit form of this polarizability is given by Eq. (2.8).
Equation (2.6) emerges by repeating the procedure with reference to Fig. 5, also allowing for the interchange of $A$ and $B$. Finally a summation of all contributory quantum amplitudes is effected.
[1] A. Ashkin, Proc. Natl. Acad. Sci. U.S.A. 94, 4853 (1997).

[2] J. E. Molloy and M. J. Padgett, Contemp. Phys. 43, 241 (2002).

[3] K. Svoboda and S. M. Block, Annu. Rev. Biophys. Biomol. Struct. 23, 247 (1994).

[4] V. I. Balykin, V. G. Minogin, and V. S. Letokhov, Rep. Prog. Phys. 63, 1429 (2000).

[5] T. A. Nieminen, H. Rubinsztein-Dunlop, and N. R. Heckenberg, J. Quant. Spectrosc. Radiat. Transf. 70, 627 (2001).

[6] D. G. Grier, Nature (London) 424, 810 (2003).

[7] A. V. Kachynski, A. N. Kuzmin, and P. N. Prasad, Proc. SPIE 5736, 10 (2005).

[8] S. J. W. Parkin, G. G. Knöner, T. A. Nieminen, N. R. Heckenberg, and H. Rubinsztein-Dunlop, Proc. SPIE 5736, 59 (2005).

[9] S. Chávez-Cerda, M. J. Padgett, I. Allison, G. H. C. New, J. C. Gutiérrez-Vega, A. T. O'Neil, I. MacVicar, and J. Courtial, J. Opt. B: Quantum Semiclassical Opt. 4, S52 (2002).

[10] E. J. Galvez, P. R. Crawford, H. I. Sztul, M. J. Pysher, P. J. Haglin, and R. E. Williams, Phys. Rev. Lett. 90, 203901 (2003).

[11] N. B. Simpson, K. Dholakia, L. Allen, and M. J. Padgett, Opt. Lett. 22, 52 (1997).

[12] T. A. Nieminen, N. R. Heckenberg, and H. RubinszteinDunlop, J. Mod. Opt. 48, 405 (2001).

[13] L. Paterson, M. P. MacDonald, J. Arlt, W. Sibbett, P. E. Bryant, and K. Dholakia, Science 292, 912 (2001).

[14] M. P. MacDonald, L. Paterson, K. Volke-Sepulveda, J. Arlt, W.
Sibbett, and K. Dholakia, Science 296, 1101 (2002).

[15] D. L. Andrews and D. S. Bradshaw, Opt. Lett. 30, 783 (2005).

[16] T. Thirunamachandran, Mol. Phys. 40, 393 (1980).

[17] D. L. Andrews and M. J. Harlow, Phys. Rev. A 29, 2796 (1984).

[18] P. W. Milonni and M. L. Shih, Phys. Rev. A 45, 4241 (1992).

[19] F. Depasse and J.-M. Vigoureux, J. Phys. D 27, 914 (1994).

[20] P. W. Milonni and A. Smith, Phys. Rev. A 53, 3484 (1996).

[21] P. C. Chaumet and M. Nieto-Vesperinas, Phys. Rev. B 64, 035422 (2001).

[22] M. Nieto-Vesperinas, P. C. Chaumet, and A. Rahmani, Philos. Trans. R. Soc. London, Ser. A 362, 719 (2004).

[23] G. Whitesides, Angew. Chem., Int. Ed. Engl. 29, 1209 (1990).

[24] M. M. Burns, J.-M. Fournier, and J. A. Golovchenko, Phys. Rev. Lett. 63, 1233 (1989).

[25] S. K. Mohanty, J. T. Andrews, and P. K. Gupta, Opt. Express 12, 2746 (2004).

[26] D. H. J. O’Dell, S. Giovanazzi, G. Kurizki, and V. M. Akulin, Phys. Rev. Lett. 84, 5687 (2000).

[27] S. Giovanazzi, D. H. J. O’Dell, and G. Kurizki, Phys. Rev. A 63, 031603 (2001).

[28] S. Giovanazzi, D. H. J. O’Dell, and G. Kurizki, J. Phys. B 34, 4757 (2001).

[29] S. Giovanazzi, D. H. J. O’Dell, and G. Kurizki, Phys. Rev. Lett. 88, 130402 (2002)

[30] I. E. Mazets, D. H. J. O’Dell, G. Kurizki, N. Davidson, and W. P. Schleich, J. Phys. B 37, S155 (2004).

[31] L. C. Dávila Romero, D. L. Andrews, and M. Babiker, J. Opt. 
B: Quantum Semiclassical Opt. 4, S66 (2002).

[32] D. P. Craig and T. Thirunamachandran, Molecular Quantum Electrodynamics (Dover, Mineola, NY, 1998).

[33] R. G. Woolley, Proc. R. Soc. London, Ser. A 456, 1803 (2000).

[34] D. L. Andrews and D. S. Bradshaw, Eur. J. Phys. 25, 845 (2004)

[35] R. D. Jenkins, D. L. Andrews, and L. C. Dávila Romero, J. Phys. B 35, 445 (2002).

[36] P. Allcock, R. D. Jenkins, and D. L. Andrews, Phys. Rev. A 61, 023812 (2000).

[37] L. Dávila Romero, S. Naguleswaran, G. E. Stedman, and D. L. Andrews, Mol. Cryst. Liq. Cryst. Sci. Technol., Sect. B: Nonlinear Opt. 23, 191 (2000).

[38] G. J. Daniels, R. D. Jenkins, D. S. Bradshaw, and D. L. Andrews, J. Chem. Phys. 119, 2264 (2003).

[39] D. L. Andrews and P. Allcock, Optical Harmonics in Molecular Systems (Wiley-VCH, Weinheim, 2002).

[40] C. G. Gray and K. E. Gubbins, Theory of Molecular Fluids (Clarendon Press, Oxford, 1984), p. 545.

[41] L. Allen, M. J. Padgett and M. Babiker, Prog. Opt. 39, 291 (1999).

[42] L. Allen, S. M. Barnett, and M. J. Padgett, Optical Angular Momentum (IOP, Bristol, 2003).

[43] A Alexandrescu, E Di Fabrizio, and D. Cojoc, e -print quant$\mathrm{ph} / 0412110$.

[44] J. E. Curtis, B. A. Koss and D. G. Grier, Opt. Commun. 207, 169 (2002).

[45] C.-H. Sun, L.-C. Yin, F. Li, G.-Q. Lu, and H.-M. Cheng, Chem. Phys. Lett. 403, 343 (2005).

[46] G. Cao, Nanostructures and Nanomaterials: Synthesis, Properties and Applications (Imperial College Press, London, 2004), p 238.

[47] M. Dequesnes, S. V. Rotkin, and N. R. Aluru, Technical Proceedings of the 2002 International Conference on Computational Nanoscience and Nanotechnology, Nanotech 2002 (unpublished) Vol. 2, p. 383.
[48] H.-J. Hwang and J.-W. Kang, Physica E (Amsterdam) 27, 163 (2005).

[49] A. Loiseau, F. Willaime, N. Demoncy, N. Schramchenko, G. Hug, C. Colliex, and H. Pascard, Carbon 36, 743 (1998).

[50] F. L. Deepak, C. P. Vinod, K. Mukhopadhyay, A. Govindaraj, and C. N. R. Rao, Chem. Phys. Lett. 353, 345 (2002).

[51] A. Gindulyte, W. N. Lipscomb, and L. Massa, Inorg. Chem. 37, 6544 (1998).

[52] I. Boustani, A. Quandt, E. Hernández, and A. Rubio, J. Chem. Phys. 110, 3176 (1999).

[53] D. Ciuparu, R. F. Klie, Y. Zhu, and L. Pfefferle, J. Phys. Chem. B 108, 3967 (2004).

[54] V. I. Prokhorenko, D. B. Steensgaard, and A. R. Holzwarth, Biophys. J. 79, 2105 (2000).

[55] C. Didraga, J. A. Klugkist and J. Knoester, J. Phys. Chem. B 106, 11474 (2002).

[56] C. Didraga, A. Pugžlys, P. R. Hania, H. Von Berlepsch, K. Duppen, and J. Knoester, J. Phys. Chem. B 108, 14976 (2004).

[57] P. N. Butcher and D. Cotter, The Elements of Nonlinear Optics (Cambridge University Press, Cambridge, U.K., 1990), p. 83.

[58] G. Juzeliūnas, Phys. Rev. A 53, 3543 (1996).

[59] G. Juzeliūnas and D. L. Andrews, Adv. Chem. Phys. 112, 357 (2000).

[60] C. A. Sackett, H. T. C. Stoof, and R. G. Hulet, Phys. Rev. Lett. 80, 2031 (1998).

[61] J. M. Gerton, D. Strekalov, I. Prodan, and R. G. Hulet, Nature (London) 408, 692 (2000).

[62] D. J. Heinzen, R. Wynar, P. D. Drummond, and K. V. Kheruntsyan, Phys. Rev. Lett. 84, 5029 (2000).

[63] F. Dimer de Oliveira and M. K. Olsen, Opt. Commun. 234, 235 (2004).

[64] R. Wynar, R. S. Freeland, D. J. Han, C. Ryu, and D. J. Heinzen, Science 287, 1016 (2000).

[65] M. Greiner, C. A. Regal, and D. S. Jin, Nature (London) 426, 537 (2003).

[66] J. Oulette, Ind. Phys. 9, 14 (2003).

[67] K. Ladavac and D. G. Grier, Opt. Express 12, 1144 (2004). 